\title{
A carbohydrate-derived trifunctional scaffold for medicinal chemistry library synthesis
}

\author{
Diana I. S. P. Resende ${ }^{1}$, Amalia M. Estévez ${ }^{1}$, André M. Alker ${ }^{2}$, Rainer E. Martin ${ }^{3, *}$ and \\ Hans Peter Wessel ${ }^{1, *}$ \\ ${ }^{1}$ Department of Chemistry, University of Aveiro, 3810-193 Aveiro, Portugal \\ ${ }^{2}$ Solid State Research, Roche Pharma Research and Early Development (pRED), Roche Innovation Center \\ Basel, F. Hoffmann-La Roche Ltd, Grenzacherstrasse 124, CH-4070 Basel, Switzerland \\ ${ }^{3}$ Medicinal Chemistry, Roche Pharma Research and Early Development (pRED), Roche Innovation Center \\ Basel, F. Hoffmann-La Roche Ltd, Grenzacherstrasse 124, CH-4070 Basel, Switzerland
}

\begin{abstract}
For the generation of compound libraries for drug discovery a central scaffold containing three exit vectors with defined chirality was devised starting from commercially available tri- $O$-acetyl-glucal. Surprisingly, the reaction of a 4- $O$-mesylate with sodium azide did not lead to the expected 4-azido-4-deoxy derivative but to a 3-azido-3-deoxy regioisomer via intermediate epoxide formation. The absolute stereochemical configuration of the final tetrahydropyran building block was proven by X-ray crystallography. This scaffold endowed with a carboxylic acid, a secondary alcohol, and an azide functionality may be connected to a DNA tag at any of the three distinct exit vectors, thus providing ready access to several different compound libraries.
\end{abstract}

Keywords: medicinal chemistry; carbohydrates; DNA; encoded libraries.

\section{Introduction}

In the search for pharmacologically interesting lead compounds, high throughput screening (HTS) is one of the most widely employed methods to scan and analyze compound libraries. In recent years DNA-encoded library technology has attracted significant attention from both industry and academia ${ }^{1}$. Using this technology all screening compounds are equipped with a unique DNA tag, and the whole library is interrogated against a therapeutic target. This is particularly useful when only small amounts of target proteins are available. In contrast to the classical HTS screening where discrete compounds are individually screened, DNAencoded libraries contain a combinatorial mixture of billions of compounds and enlarging the size of the library incurs only minimal additional costs upon screening ${ }^{2}$. Franzini and Randolph ${ }^{3}$ have investigated the chemical space and topologies of DNA encoded chemical libraries, and central scaffold used were only triazines ${ }^{2,4}$ and simply hydroxy- ${ }^{5}$ or aminoproline ${ }^{6}$. We have recently presented a carbohydrate derived central scaffold for use in DNA-encoded library technology 7 . While triazines are flat and thus medicinally less interesting, the pyran scaffold is conformationally rather rigid and can present its residues in a defined three-dimensional orientation. Carbohydrates with

*Corresponding author: Hans Peter Wessel

Email address: hanspwessel11@gmail.com

DOI: http://dx.doi.org/10.13171/mjc72/01809051415-wessel their pre-existing conformationally defined substituents are ideally suited, and herein we discuss a further example starting from glucal.

\section{Results and Discussion}

Commercial tri- $O$-acetyl-D-glucal was deacetylated with a basic ion exchange resin ${ }^{8}$, and the resulting D-glucal was directly selectively oxidized with palladium acetate and vinyl acetate as the proton acceptor to furnish enone 1 in $82 \%$ overall yield ${ }^{9}$. The decent yield of $86 \%$ reported ${ }^{10}$ for the selective reduction of the double bond of enone 1 with palladium-on-carbon in ethanol could not be reproduced. In our hands, the yields obtained for compound 2 were $<40 \%$. However, employing ethyl acetate as a solvent increased the yield to acceptable $56 \%$. The primary hydroxyl group was selectively silylated with tert-butyldiphenylsilyl chloride to give compound 3 in $93 \%$ yield. The bulky silyl protective group was chosen not only to obtain a selective silylation reaction but also to influence the stereochemical outcome ${ }^{11}$ of the reduction of the 3oxo group at a later stage. In order to simplify the chromatographic purification of the hydrogenated product, we have also inverted the reaction sequence. Diol 1 was silylated to give the tertbutyldiphenylsilyl protected enitol 4 (93\%) followed by hydrogenation to compound $\mathbf{3}$ which again was

\footnotetext{
Received July 3, 2018

Accepted, August 21, 2018

Published September 5, 201
} 
not selective and only low-yielding (37\%). Mesylation of enitol 4 afforded compound 5 in $67 \%$ yield which was hydrogenated to furnish tetrahydropyran derivative 6 in $37 \%$ yield. The identical compound was accessible from hexulose 3 by mesylation of the secondary alcohol group (Scheme
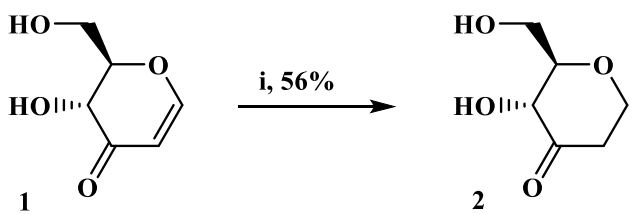

iii, $93 \%$

ii, $93 \%$

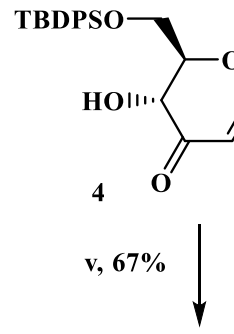<smiles>CC(C)(C)[Si](C)(c1ccccc1)c1ccccc1</smiles><smiles>CO[C@H]1C(=O)C=CO[C@@H]1CO[18OH]</smiles>

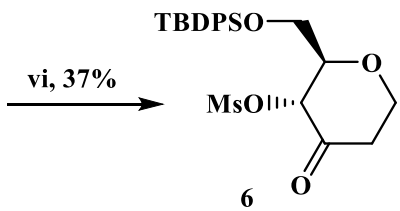

Scheme 1. Reagents and conditions: (i) $\mathrm{H}_{2}, \mathrm{Pd} / \mathrm{C}$, EtOAc, rt, $15 \mathrm{~h}$; (ii) TBDPSCl, imidazole, $\mathrm{CH}_{2} \mathrm{Cl}_{2}, \mathrm{rt}, 10 \mathrm{~min}$; (iii) TBDPSCl, Im, $\mathrm{CH}_{2} \mathrm{Cl}_{2}$, rt, $30 \mathrm{~min}$; (iv) $\mathrm{H}_{2}, \mathrm{Pd} / \mathrm{C}$, EtOH, rt, 15 h; (v) $\mathrm{MsCl}, \mathrm{Py}, 0{ }^{\circ} \mathrm{C} \rightarrow \mathrm{rt}, 4 \mathrm{~h}$; (vi) $\mathrm{H}_{2}, \mathrm{Pd} / \mathrm{C}$, AcOEt/EtOH, rt,2 h; (vii) $\mathrm{MsCl}, \mathrm{Py}, 0{ }^{\circ} \mathrm{C}->\mathrm{rt}$, 4h. TBDPS = tert-butyldiphenylsilyl.

In an attempt to improve the yield of the alkene reduction step we looked at this stage also into a less sterically hindered silyl protective group. The reaction of enone 1 with tert-butyldimethylsilyl chloride yielded the silyl ether 7 in $86 \%$. This compound had been prepared before by oxidation of the respective glucal 12,13 . While the tert- butyldimethylsilyl ether 7 is nicely crystalline, mesylate 8 was only obtained as highly viscous oil. Hydrogenation of compound 7 provided only a small amount of the anticipated 3-oxo product $9(18 \%)$, the main product was the diol $10(58 \%)$ in the favored D-arabino configuration with the newly formed hydroxyl group in equatorial position (Scheme 2).

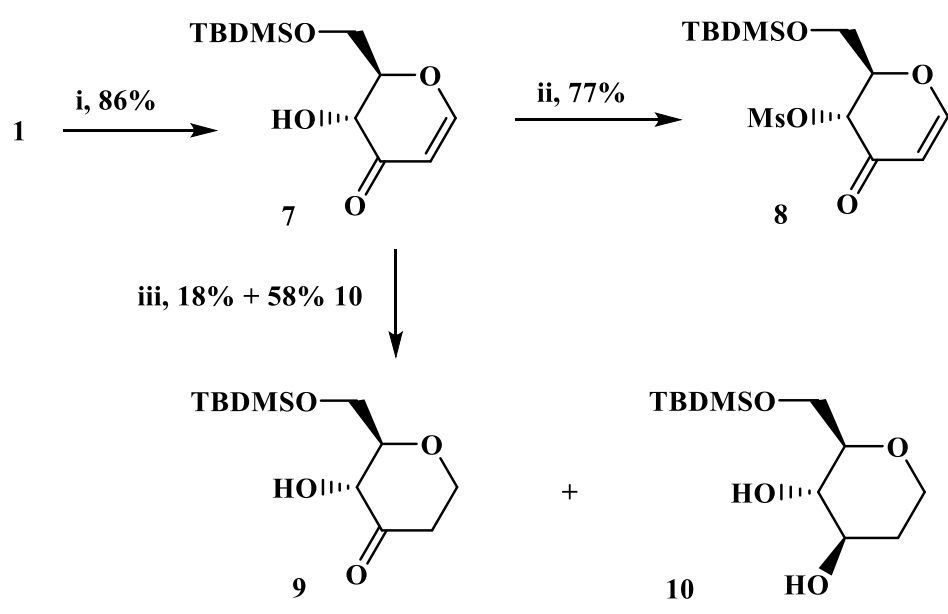

Scheme 2. Reagents and conditions: (i) TBDPMCl, imidazole, $\mathrm{CH}_{2} \mathrm{Cl}_{2}$, rt, $30 \mathrm{~min}$; (ii) $\mathrm{MsCl}, \mathrm{Py}, 0{ }^{\circ} \mathrm{C}->\mathrm{rt}, 4 \mathrm{~h}$; (iii) $\mathrm{H}_{2}, \mathrm{Pd} / \mathrm{C}, \mathrm{EtOH}, \mathrm{rt}, 20 \mathrm{~min} . \mathrm{Ms}=$ mesyl, TBDMS = tert-butyldimethylsilyl. 
In order to capitalize on the steric properties, we thus continued with the tert-butyldiphenylsilyl protected hexulose 6 reducing the oxo function with sodium borohydride to result in mainly D-arabino configured compound $11^{14}$. The ratio of D-arabino to D-ribo product 12 was $87: 13$ as determined by ${ }^{1} \mathrm{H}-\mathrm{NMR}$ integration (Scheme 3). In contrast, with an $\alpha$-anomeric substituent only the $\mathrm{D}$-allo product (analogous to D-ribo in our case) had been obtained ${ }^{15}$. Likewise, 1,5-anhydro-4,6-di- $O$-tertbutyldiphenylsilyl-2-deoxy-D-erythro-hex-1-en-3ulose had been reported ${ }^{4}$ to strongly favor the D-ribo derivative upon sodium borohydride reduction, which hints at the directing influence of a bulky substituent at $O-4$. Unfortunately, treatment of the mesylated and 6-O-silyl protected enones $\mathbf{5}$ and $\mathbf{8}$ with sodium borohydride led to major decomposition.

Next, the mesylate 11 was treated with sodium azide in DMSO in an attempt to introduce a nitrogen functionality in the 4-position. To our initial surprise the 4-position was not substituted, and instead, the azide was introduced in the 3-position as evidenced by a series of 2D-NMR experiments. In particular, a $2 \mathrm{ax} / 4-\mathrm{OH}$ crosspeak was observed in the NOESY spectrum demonstrating the inversion at the C-4 position. This experimental outcome can be explained by initial epoxide formation followed by the opening of the three-membered ring by azide at the sterically less hindered C-atom. Indeed, a closer look at the previous borohydride reduction revealed the formation of epoxide $\mathbf{1 3}$ as a minor by-product (9\%) next to the formation of the desired compounds 11 and 12. Finally, the 3 -azido derivative 14 was silyl deprotected to furnish the diol 15 which was then selectively oxidized at the primary alcohol position in a two-step process employing TEMPO and subsequently sodium chlorite oxidation ${ }^{16}$ to give the final scaffold 16. The absolute stereochemical configuration of the trifunctional scaffold $\mathbf{1 6}$ was unequivocally proven by single crystal X-ray diffraction analysis (Figure 1) ${ }^{17}$.

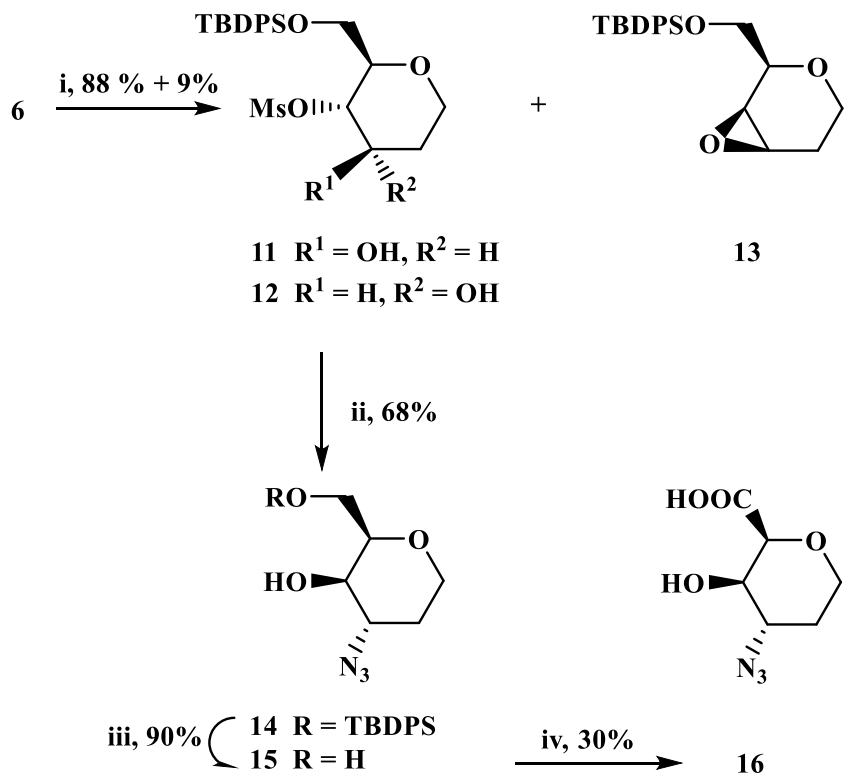

Scheme 3. Reagents and conditions: (i) $\mathrm{NaBH}_{4}, \mathrm{EtOH} / \mathrm{H}_{2} \mathrm{O}, \mathrm{rt}, 10 \mathrm{~min}$; (ii) $\mathrm{NaN}_{3}, \mathrm{DMF}, 90{ }^{\circ} \mathrm{C}, 2$ days; (iii) tert$\mathrm{Bu}_{4} \mathrm{NF}$, THF, rt, 1h; (iv) tert-BuNI, tetramethylpiperidine- $N$-oxide (TEMPO), (diacetoxyiodo)benzene (BAIB), $\mathrm{CH}_{2} \mathrm{Cl}_{2} / \mathrm{H}_{2} \mathrm{O}$ (3:1), then 2-methyl-2-butene, $\mathrm{NaClO}_{2}, \mathrm{NaH}_{2} \mathrm{PO}_{4}, \mathrm{H}_{2} \mathrm{O}, 1 \mathrm{~h}$.

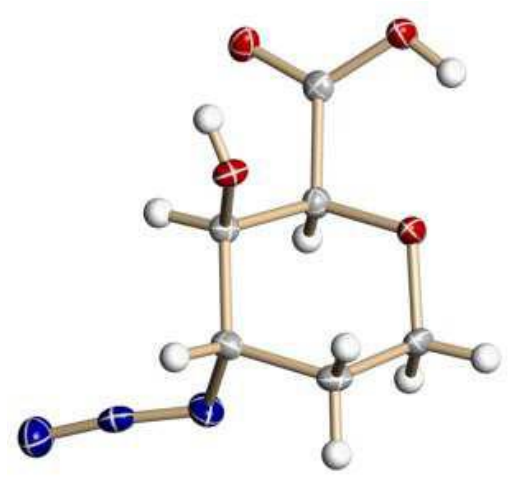

Figure 1. Single crystal X-ray diffraction analysis of azide 16. The ORTEP drawing depicts thermal ellipsoids at a $30 \%$ probability level. 


\section{Conclusion}

In summary, we have demonstrated the synthesis of a trifunctional scaffold endowed with a carboxylic acid, a secondary alcohol and an azide functionality for use as a non-planar building block in medicinal chemistry library synthesis aimed at drug discovery. Furthermore, this building block is ideally suited for attachment of a DNA tag via any of the functional groups. The scaffold can then be expanded at the two remaining vectors, thus, enabling the generation of several DNA-encoded libraries. The azide, as a masked amino group, inherently carries a double function as it can be either reacted with acetylenes in a click-type reaction to triazoles or after reduction to a primary amino group can undergo the usual amine type reactions such as amide or sulfonamide formation. Libraries generated from this building block contain a 3-amino-3-deoxy sugar-type derivative as a core, which is a prominent feature in numerous biologically active naturally derived compounds. Alternatively, when considering the corresponding amino group, this core may be viewed as a conformationally restrained $\gamma$-amino acid that can mimic biologically relevant exit vectors in proteins.

\section{Acknowledgements}

This work was supported by a Hoffmann-La Roche Fellowship (RPF-ID 297) for A.M.E. The authors would like to thank Dr. Inken Plitzko and $\mathrm{Mr}$. Markus Bürkler (NMR), Ms. Sophie Brogly (MS), and Mr. Daniel Zimmerli (optical rotations).

\section{Experimental}

\section{General Methods}

Most reagents were purchased from Sigma Aldrich, palladium-on-carbon (10\%) and toluenesulfonyl chloride from Merck. Solvents were purchased from either Fischer-Scientific, VWR or Lab-Scan and used without further purification unless otherwise stated, $N, N^{\prime}$-dimethylformamide (DMF) and pyridine were dried over $4 \AA$ molecular sieves. Analytical thin layer chromatography (TLC) was performed on was performed by using $20 \times 20 \mathrm{~cm}$ ALUGRAM® SIL G aluminum sheets from Macherey-Nagel $\mathrm{GmbH} \&$ Co. KG, and the TLC spots were visualized with Hanessian mixture. Medium pressure liquid chromatography (MPLC) was performed on an Isco Combiflash Rf200 system using pre-packed normal phase disposable columns for flash chromatography.

Melting points were determined on a Büchi Melting Point B-540 apparatus. ${ }^{1} \mathrm{H}$ Nuclear Magnetic Resonance (NMR) spectra were recorded on a Bruker Avance III $600 \mathrm{MHz}$ spectrometer. In the ${ }^{1} \mathrm{H}$
NMR spectra, signal positions $(\delta)$ are given in parts per million $(\mathrm{ppm})$ from tetramethylsilane $(\delta=0)$ and were measured relative to the signal of $\mathrm{CDCl}_{3}$ $(\delta=7.26 \mathrm{ppm})$, DMSO-d $\mathrm{d}_{6}(2.50 \mathrm{ppm})$ or $\mathrm{CD}_{3} \mathrm{OD}$ (3.31 ppm). Resonances in the ${ }^{1} \mathrm{H}$ NMR spectra are reported to the nearest $0.01 \mathrm{ppm} .{ }^{13} \mathrm{C}$ NMR spectra were recorded using the same spectrometer (150 $\mathrm{MHz}$ ), and signal positions $(\delta)$ are given in parts per million $(\mathrm{ppm})$ from tetramethylsilane $(\delta=0)$ and were measured relative to the signal of $\mathrm{CDCl}_{3}$ ( $\delta=77.16 \mathrm{ppm})$, DMSO-d 6 (39.52 ppm) or $\mathrm{CD}_{3} \mathrm{OD}$ $(49.00 \mathrm{ppm})$ and reported to the nearest $0.1 \mathrm{ppm}$. HSQC-DEPT Spectra were measured routinely. Unequivocal assignments were made with the aid of 2D HMBC, COSY, and NOESY experiments. The multiplicities of signals are given as $\mathrm{s}=$ singlet, $\mathrm{d}=$ doublet, $\mathrm{t}=$ triplet, $\mathrm{q}=$ quartet, quin = quintet, $\mathrm{m}=$ multiplet, $\mathrm{br}=$ broadened and combinations thereof. Coupling constants $(\mathrm{J})$ are reported to the nearest $0.1 \mathrm{~Hz}$. Anomeric carbons were numbered 1 . High resolution mass spectrometry (HRMS) was performed on a Finnigan LTQ FT MS or an Agilent 6520 spectrometer using time of flight (TOF) with positive ESI at $70 \mathrm{eV}$ within a tolerance of $\pm 5 \mathrm{ppm}$ of the theoretical value.

Data for 1,5-Anhydro-2-deoxy-D-erythro-hex-1-en3-ulose (1) ${ }^{9}$

${ }^{1} \mathrm{H}$ NMR (600 MHz, methanol-d $\mathrm{d}_{4}$, HSQC, HMBC) $\delta 7.55$ (slightly broadened $\mathrm{d}, 1 \mathrm{H}, \mathrm{H}-1$ ), 5.40 $\left(\mathrm{d}, J_{1,2}=5.8 \mathrm{~Hz}, 1 \mathrm{H}, \mathrm{H}-2\right), 4.35\left(\mathrm{~d}, J_{4,5}=13.0 \mathrm{~Hz}\right.$, $1 \mathrm{H}, \mathrm{H}-4$ ), 4.18 (dddd, $J_{1,5}=0.7 \mathrm{~Hz}, 1 \mathrm{H}, \mathrm{H}-5$ ), 4.01 $\left(\mathrm{dd}, J_{5,6 \mathrm{a}}=2.2 \mathrm{~Hz}, J_{6 \mathrm{a}, 6 \mathrm{a}}=12.6 \mathrm{~Hz}, 1 \mathrm{H}, \mathrm{H}-6 \mathrm{a}\right), 3.90$ $\left(\mathrm{dd}, J_{5,6 \mathrm{~b}}=4.4 \mathrm{~Hz}, J_{6 \mathrm{a}, 6 \mathrm{a}}=12.6 \mathrm{~Hz}, 1 \mathrm{H}, \mathrm{H}-6 \mathrm{~b}\right)$;

${ }^{1} \mathrm{H}$ NMR (600 MHz, $\mathrm{CDCl}_{3}$, HSQC-DEPT) $\delta 7.43$ (slightly broadened d, $1 \mathrm{H}, \mathrm{H}-1), 5.50\left(\mathrm{~d}, J_{1,2}=5.9\right.$ $\mathrm{Hz}, 1 \mathrm{H}, \mathrm{H}-2), 4.36$ (d, $\left.J_{4,5}=13.3 \mathrm{~Hz}, 1 \mathrm{H}, \mathrm{H}-4\right), 4.19$ (dddd, $\left.J_{1,5}=0.6 \mathrm{~Hz}, 1 \mathrm{H}, \mathrm{H}-5\right), 4.12\left(\mathrm{dd}, J_{5,6 \mathrm{a}}=2.7\right.$ $\left.\mathrm{Hz}, J_{6 \mathrm{a}, 6 \mathrm{a}}=12.6 \mathrm{~Hz}, 1 \mathrm{H}, \mathrm{H}-6 \mathrm{a}\right), 4.02\left(\mathrm{dd}, J_{5,6 \mathrm{~b}}=4.4\right.$ $\left.\mathrm{Hz}, J_{6 \mathrm{a}, 6 \mathrm{a}}=12.6 \mathrm{~Hz}, 1 \mathrm{H}, \mathrm{H}-6 \mathrm{~b}\right)$.

Data for 1,5-Anhydro-2-deoxy-D-erythro-hex-3ulose (2) 10

${ }^{1} \mathrm{H}$ NMR (600 MHz, methanol-d $\mathrm{d}_{4}$, HSQC-DEPT, HSQC-HMBC, COSY) $\delta 4.28$ (ddd, $J_{1 \text { ax }, \text { leq }}=11.3$ $\left.\mathrm{Hz}, J_{\text {leq,2ax }}=7.5 \mathrm{~Hz}, J_{\text {leq,2eq }}=1.2 \mathrm{~Hz}, 1 \mathrm{H}, \mathrm{H}-1_{\mathrm{eq}}\right)$, $4.14\left(\mathrm{dd}, J_{4,5}=10.0 \mathrm{~Hz}, J_{2 \mathrm{ax}, 4}=1.2 \mathrm{~Hz}, 1 \mathrm{H}, \mathrm{H}-4\right)$, $3.88\left(\mathrm{dd}, J_{5,6 \mathrm{a}}=2.1 \mathrm{~Hz}, J_{6 \mathrm{a}, 6 \mathrm{~b}}=12.0 \mathrm{~Hz}, 1 \mathrm{H}, \mathrm{H}-6 \mathrm{a}\right)$, $3.76\left(\mathrm{dd}, J_{5,6 \mathrm{~b}}=5.1 \mathrm{~Hz}, J_{6 \mathrm{a}, 6 \mathrm{~b}}=12.0 \mathrm{~Hz}, 1 \mathrm{H}, \mathrm{H}-6 \mathrm{~b}\right)$, $3.65\left(\mathrm{ddd}, J_{1 \mathrm{ax}, \text { leq }}=11.3 \mathrm{~Hz}, J_{1 \mathrm{ax}, 2 \mathrm{ax}}=12.6 \mathrm{~Hz}\right.$, $\left.J_{1 \text { ax, 2eq }}=2.5 \mathrm{~Hz}, 1 \mathrm{H}, \mathrm{H}-2 \mathrm{ax}\right), 3.34\left(\mathrm{ddd}, J_{4,5}=10.0\right.$ $\left.\mathrm{Hz}, J_{5,6 \mathrm{a}}=2.1 \mathrm{~Hz}, J_{5,6 \mathrm{~b}}=5.1 \mathrm{~Hz}, 1 \mathrm{H}, \mathrm{H}-5\right), 2.80$ $\left(\right.$ dddd, $J_{\text {leq }, 2 \mathrm{ax}}=7.5 \mathrm{~Hz}, \quad J_{1 \mathrm{ax}, 2 \mathrm{ax}}=12.6 \mathrm{~Hz}$, $\left.J_{2 \mathrm{ax}, 2 \mathrm{eq}}=14.0 \mathrm{~Hz}, J_{2 \mathrm{ax}, 4}=1.5 \mathrm{~Hz}, 1 \mathrm{H}, \mathrm{H}-2 \mathrm{ax}\right), 2.41$ $\left(\mathrm{ddd}, J_{1 \mathrm{ax}, 2 \mathrm{eq}}=2.5 \mathrm{~Hz}, J_{1 \mathrm{eq}, 2 \mathrm{eq}}=1.2 \mathrm{~Hz}, J_{2 \mathrm{ax}, 2 \mathrm{eq}}=14.0\right.$ $\mathrm{Hz}, 1 \mathrm{H}, \mathrm{H}-2 \mathrm{eq})$;

${ }^{13} \mathrm{C}$ NMR (151 MHz, methanol-d 4 ) $\delta 210.0$ (C-3), 86.6 (C-5), 76.0 (C-4), 69.3 (C-1), 64.1 (C-6), 43.5 (C-2). 


\section{1,5-Anhydro-6-O-tert-butyldiphenylsilyl-2-deoxy- D-erythro-hex-1-en-3-ulose (3)}

Method A. To a solution of compound 2 (0.29 g, $1.98 \mathrm{mmol})$ in dichloromethane $(4 \mathrm{~mL})$ were added $t$-butyldiphenylchlorosilane $(0.71 \mathrm{~g}, 2.57 \mathrm{mmol})$ and imidazole $(0.34 \mathrm{~g}, 4.95 \mathrm{mmol})$. The reaction mixture was stirred at $\mathrm{rt}$ for $30 \mathrm{~min}$. After this time TLC (ethyl acetate) showed the complete consumption of the starting material and the formation of one major product. The reaction mixture was washed with water $(5 \mathrm{~mL})$, and the organic layer was dried (sodium sulfate) and concentrated under reduced pressure. The resulting residue was purified by column chromatography (ethyl acetate/hexane 1:5) to give silylated compound $3(0.70 \mathrm{~g}, 93 \%)$ as a colorless oil.

Method B. Through a solution of compound 4 $(11.14 \mathrm{~g}, 29.1 \mathrm{mmol})$ in ethanol $(400 \mathrm{~mL})$ was bubbled argon for five minutes. Palladium-on-carbon $(10 \%, 557 \mathrm{mg}, 5 \% \mathrm{wt})$ was added carefully. The reaction flask was evacuated, and hydrogen was added. The mixture was stirred for 15 hours when complete removal of starting material was detected (ethyl acetate). The catalyst was filtered off through celite, and the solvent was reduced in vacuo. The resulting crude oil was subjected to column chromatography (ethyl acetate) to furnish compound $3(4.154 \mathrm{~g}, 37 \%)$ as a colorless oil: $[\alpha]_{20}^{\mathrm{D}}=+34.4$ (c=1.0 in methanol)

${ }^{1} \mathrm{H}$ NMR (600 MHz, DMSO-d $\mathrm{d}_{6}$, HSQC-DEPT, COSY, NOESY) $\delta 7.70-7.66(\mathrm{~m}, 8 \mathrm{H}, \mathrm{Ph}), 7.47-7.38$ $(\mathrm{m}, 6 \mathrm{H}, \mathrm{Ph}), 5.32\left(\mathrm{~d}, J_{4,4-\mathrm{OH}}=5.6 \mathrm{~Hz}, 1 \mathrm{H}, 4-\mathrm{OH}\right)$, $4.21\left(\mathrm{ddd}, J_{1 \mathrm{ax}, \text { leq }}=11.2 \mathrm{~Hz}, J_{\text {leq, } 2 \mathrm{ax}}=7.5 \mathrm{~Hz}\right.$, $\left.J_{1 \text { eq, 2eq }}=1.1 \mathrm{~Hz}, 1 \mathrm{H}, \mathrm{H}-1 \mathrm{eq}\right), 4.14$ (ddd, $J_{2 \mathrm{ax}, 4}=1.2$ $\left.\mathrm{Hz}, J_{4,4-\mathrm{OH}}=5.6 \mathrm{~Hz}, J_{4,5}=9.9 \mathrm{~Hz}, 1 \mathrm{H}, \mathrm{H}-4\right), 3.93(\mathrm{dd}$ $\left.J_{5,6 \mathrm{a}}=1.6 \mathrm{~Hz}, J_{6 \mathrm{a}, 6 \mathrm{~b}}=11.1 \mathrm{~Hz}, 1 \mathrm{H}, \mathrm{H}-6 \mathrm{a}\right), 3.88(\mathrm{dd}$, $J_{5,6 \mathrm{~b}}=4.8 \mathrm{~Hz}, J_{6 \mathrm{a}, 6 \mathrm{~b}}=11.1 \mathrm{~Hz}, 1 \mathrm{H}, \mathrm{H}-6 \mathrm{~b}$ ), 3.58 (ddd, $J_{1 \mathrm{ax}, 1 \mathrm{eq}}=11.3 \mathrm{~Hz}, J_{1 \mathrm{ax}, 2 \mathrm{ax}}=12.6 \mathrm{~Hz}, J_{1 \mathrm{ax}, 2 \mathrm{eq}}=2.4 \mathrm{~Hz}$, $1 \mathrm{H}, \mathrm{H}-1 \mathrm{ax}$ ), 3.42 (ddd, $J_{4,5}=9.9 \mathrm{~Hz}, J_{5,6 \mathrm{a}}=1.7 \mathrm{~Hz}$, $\left.J_{5,6 \mathrm{~b}}=4.8 \mathrm{~Hz}, 1 \mathrm{H}, \mathrm{H}-5\right), 2.76$ (dddd, $J_{\text {leq, 2ax }}=7.5 \mathrm{~Hz}$, $\left.J_{2 \mathrm{ax}, \text { 2eq }}=14.1 \mathrm{~Hz}, 1 \mathrm{H}, \mathrm{H}-2 \mathrm{ax}\right), 2.31(\mathrm{ddd}, 1 \mathrm{H}$, $\mathrm{H}-2 \mathrm{eq}), 1.01$ (s, 9H, $t \mathrm{Bu}$ );

${ }^{13} \mathrm{C}$ NMR (151 MHz, DMSO-d 6$) \delta 199.7(\mathrm{C}=\mathrm{O})$, $135.1(2 \mathrm{C}, o \mathrm{Ph}), 134.4(2 \mathrm{C}, o \mathrm{Ph}), 133.1$ (2C, $\mathrm{Si}-\mathrm{CPh}), 129.2(2 \mathrm{C}, p \mathrm{Ph}), 127.8(2 \mathrm{C}, m \mathrm{Ph}), 127.5$ (2C, $m \mathrm{Ph}), 83.4$ (C-5), $73.3(\mathrm{C}-4), 66.3$ (C-1), 64.0 (C-6), 41.3 (C-2), 26.7 (3C, $\left.\mathrm{CH}_{3}\right), 19.0$ (Si-C); MS (IS pos.) $m / z$ 402. $1\left(\mathrm{M}+\mathrm{NH}_{4}^{+}\right), 437.1\left(\mathrm{M}+\mathrm{Na}^{+}\right)$.

\section{1,5-Anhydro-6-O-tert-butyldiphenylsilyl-2-deoxy- D-erythro-hex-1-en-3-ulose (4)}

To a solution of compound 1 (4.53 g, $31.42 \mathrm{mmol})$ in dichloromethane $(60 \mathrm{~mL})$ were added $t$-butyldiphenylchlorosilane $(9.0 \mathrm{~mL}, 35.56 \mathrm{mmol})$ and imidazole $(5.35 \mathrm{~g}, 78.55 \mathrm{mmol})$. The reaction mixture was stirred at room temperature for $10 \mathrm{~min}$. After this time TLC (ethyl acetate) showed the complete consumption of the starting material and the formation of one major product. The reaction mixture was washed with water $(5 \mathrm{~mL})$, and the organic layer was dried over sodium sulfate and concentrated under reduced pressure. The resulting residue was purified by column chromatography (ethyl acetate/hexane 1:5) to give pure compound 4 $(11.22 \mathrm{~g}, 93 \%)$ as a colorless oil: $[\alpha]^{\mathrm{D}}{ }_{20}=+131.7(c$ 1.0 in methanol);

${ }^{1} \mathrm{H}$ NMR (300 MHz, DMSO-d ${ }_{6}$, HSQC, HMBC, NOESY) $\delta$ 7.68-7.63 (m, 5H, H-1, o-Ph), 7.49-7.38 $(\mathrm{m}, 6 \mathrm{H}, m-\mathrm{Ph}, p-\mathrm{Ph}), 5.81\left(\mathrm{~d}, J_{4,4-\mathrm{OH}}=4.9 \mathrm{~Hz}, 1 \mathrm{H}\right.$, $4-\mathrm{OH}), 5.33\left(\mathrm{~d}, J_{1,2}=5.8 \mathrm{~Hz}, 1 \mathrm{H}, \mathrm{H}-2\right), 4.31\left(\mathrm{dd}, J_{4,5}\right.$ $\left.=12.4 \mathrm{~Hz}, J_{4,4-\mathrm{OH}}=4.9 \mathrm{~Hz}, 1 \mathrm{H}, \mathrm{H}-4\right), 4.23(\mathrm{ddd} \approx \mathrm{dt}$, 1H, H-5), 4.06-3.93 (m, 2H, H-6a, H-6b), 0.99 (s, $9 \mathrm{H}, t \mathrm{Bu}) ;{ }^{1} \mathrm{H} \mathrm{NMR}\left(600 \mathrm{MHz}, \mathrm{CDCl}_{3}, \mathrm{HSQC}-\right.$ DEPT) $\delta 7.73-7.70(\mathrm{~m}, 4 \mathrm{H}, o-\mathrm{Ph}), 7.45$ - 7.38 $(\mathrm{m}, 7 \mathrm{H}, m-\mathrm{Ph}, p-\mathrm{Ph}, \mathrm{H}-1), 5.47$ (d, $J_{1,2}=5.7 \mathrm{~Hz}, 1 \mathrm{H}$, $\mathrm{H}-2), 4.51$ (dd, $\left.J_{4,5}=13.2 \mathrm{~Hz}, 1 \mathrm{H}, \mathrm{H}-4\right), 4.16$ (ddd, $1 \mathrm{H}, \mathrm{H}-5), 4.10\left(\mathrm{dd}, J_{5,6 \mathrm{a}}=2.0 \mathrm{~Hz}, J_{6 \mathrm{a}, 6 \mathrm{~b}}=11.9 \mathrm{~Hz}\right.$, $1 \mathrm{H}, \mathrm{H}-6 \mathrm{a}), 4.06\left(\mathrm{dd}, J_{5,6 \mathrm{~b}}=3.6 \mathrm{~Hz}, J_{6 \mathrm{a}, 6 \mathrm{~b}}=11.9 \mathrm{~Hz}\right.$, $1 \mathrm{H}, \mathrm{H}-6 \mathrm{~b}), 3.49$ (d, $\left.J_{4,4-\mathrm{OH}}=1.3 \mathrm{~Hz}, 1 \mathrm{H}, 4-\mathrm{OH}\right), 1.03$ - $1.08(\mathrm{~m}, 9 \mathrm{H}, t \mathrm{Bu}) ;{ }^{13} \mathrm{C}$ NMR (75 MHz, DMSO-d $\left.\mathrm{d}_{6}\right)$ $\delta 194.4(\mathrm{C}=\mathrm{O}), 163.7(\mathrm{C}-1), 135.2(4 \mathrm{C}, o-\mathrm{Ph}), 132.8$ (2C, Si-CPh), 130.0 (2C, $p-\mathrm{Ph}), 128.0$ (4C, $m-\mathrm{Ph})$, 104.0 (C-2), 82.9 (C-5), 67.5 (C-4), 62.6 (C-6), 26.7 (3C, $\left.\mathrm{CH}_{3}\right), 19.0(\mathrm{Si}-\mathrm{C})$;

${ }^{13} \mathrm{C}$ NMR (151 MHz, $\left.\mathrm{CDCl}_{3}\right) \delta 193.7(\mathrm{C}=\mathrm{O}), 163.6$ (C-1), 134.8 (4C, oPh), 135.4 (2C, Si-CPh), 132.3 $(p-\mathrm{Ph}), 132.2(p-\mathrm{Ph}), 128.9$ (2C, $m-\mathrm{Ph}), 126.89$, 126.88 (2C, $m-\mathrm{Ph}), 102.3$ (C-2), 82.6 (C-5), 66.5 (C-4), 61.5 (C-6), 25.9 (3C, $\left.\mathrm{CH}_{3}\right), 18.5$ (Si-C) ;

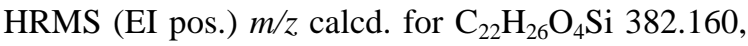
found 382.159

\section{1,5-Anhydro-6-O-tert-butyldiphenylsilyl-4-O- methanesulphonyl-2-deoxy-D-erythro-hex-1-en-3- ulose (5)}

To a solution of compound 4 (3.92 g, $10.26 \mathrm{mmol})$ in dry pyridine $(20 \mathrm{~mL})$ was added methanesulfonyl chloride $(0.87 \mathrm{~mL}, 11.28 \mathrm{mmol})$ at $0{ }^{\circ} \mathrm{C}$. The reaction mixture was stirred for $4 \mathrm{~h}$ at $\mathrm{rt}$. After this time TLC (ethyl acetate/hexane 1:3) indicated the complete consumption of the starting material. The reaction mixture was concentrated under reduced pressure. The residue was taken up in ethyl acetate $(35 \mathrm{~mL})$ and washed with $10 \%$ aqueous $\mathrm{HCl}$ solution $(30 \mathrm{~mL})$ and then aqueous saturated sodium bicarbonate solution $(30 \mathrm{~mL})$. The organic layer was dried over sodium sulphate and concentrated in vacuo. The crude product was purified by column chromatography using ethyl acetate/hexane 1:5 to give the pure mesylate $5(3.09 \mathrm{~g}, 67 \%)$ as a colorless crystals, mp $105.6-106.3{ }^{\circ} \mathrm{C} ;[\alpha]^{\mathrm{D}}{ }_{20}=+149.2(\mathrm{c}=0.5$ in methanol);

${ }^{1} \mathrm{H}$ NMR (300 MHz, DMSO-d $\mathrm{d}_{6}, \mathrm{HSQC}, \mathrm{HMBC}$, COSY) $\delta 7.78\left(\mathrm{~d}, J_{1,2}=5.8 \mathrm{~Hz}, 1 \mathrm{H}, \mathrm{H}-1\right), 7.70-7.66$ $(\mathrm{m}, 4 \mathrm{H}, \mathrm{oPh}), 7.51-7.40(\mathrm{~m}, 6 \mathrm{H}, m \mathrm{Ph}, p \mathrm{Ph}), 5.51(\mathrm{~d}$, $\left.J_{1,2}=5.9 \mathrm{~Hz}, 1 \mathrm{H}, \mathrm{H}-2\right), 5.51\left(\mathrm{~d}, J_{4,5}=11.6 \mathrm{~Hz}, 1 \mathrm{H}\right.$, $\mathrm{H}-4), 4.70\left(\mathrm{ddd} \approx \mathrm{dt}, J_{4,5}=11.7 \mathrm{~Hz}, J_{5,6 \mathrm{a}}=2.4 \mathrm{~Hz}\right.$, $1 \mathrm{H}, \mathrm{H}-5)$, 4.02-3.92 (m, 2H, $J_{5,6 \mathrm{~b}}=2.4 \mathrm{~Hz}, \mathrm{H}-6 \mathrm{a}$, $\mathrm{H}-6 \mathrm{~b}), 3.38$ (s, 3H, $\left.-\mathrm{SCH}_{3}\right), 1.00(\mathrm{~s}, 9 \mathrm{H}, t \mathrm{Bu})$;

${ }^{13} \mathrm{C}$ NMR (75 MHz, DMSO-d $\left.{ }_{6}\right) \delta 187.4(\mathrm{C}=\mathrm{O})$, $164.6(\mathrm{C}-1), 135.25(2 \mathrm{C}, o \mathrm{Ph}), 135.21(2 \mathrm{C}, o \mathrm{Ph})$, 132.3 (Si-CPh), 132.2 (Si-CPh), 130.0 (2C, pPh), 
127.9 (4C, $m \mathrm{Ph}), 104.1$ (C-2), 80.1 (C-5), 73.8 (C-4), $61.6(\mathrm{C}-6), 38.7\left(\mathrm{SCH}_{3}\right), 26.5\left(3 \mathrm{C}, \mathrm{CH}_{3}\right), 18.9$ (Si-C);

HRMS (EI pos.) $m / z$ calcd. for $\mathrm{C}_{23} \mathrm{H}_{28} \mathrm{O}_{6} \mathrm{SSi} 460.138$, found 460.137 .

1,5-Anhydro-6-O-tert-butyldiphenylsilyl-2-deoxy4-O-methanesulphonyl-D-erythro-hex-3-ulose (6)

Method A. Through a solution of compound 5 ( $0.5 \mathrm{~g}$, $14.68 \mathrm{mmol}$ ) in a mixture of ethyl acetate/ethanol $(5: 1,30 \mathrm{~mL})$ was bubbled argon for five minutes. Palladium on carbon $(10 \% \mathrm{Pd} / \mathrm{C})(52 \mathrm{mg}, 5 \% \mathrm{wt})$ was added carefully. The reaction flask was evacuated, and hydrogen was added. The mixture was stirred for 2 hours when the full conversion was detected (AcOEt/hexane 3:7). The catalyst was filtered off through celite, and the solvent was reduced in vacuo. The resulting crude oil was subjected to column chromatography (AcOEt/hexane $1: 5)$ to furnish compound $6(0.188 \mathrm{~g}, 37 \%)$ as a colourless oil.

Method B. To a solution of compound 3 (0.96 g, $2.49 \mathrm{mmol})$ in dry pyridine $(4.2 \mathrm{~mL})$ was added methanesulfonyl chloride $(0.48 \mathrm{~mL}, 2.75 \mathrm{mmol})$ at $0^{\circ} \mathrm{C}$. The reaction mixture was stirred for $2 \mathrm{~h}$ at $\mathrm{rt}$. After this time TLC (ethyl acetate/hexane 1:3) indicated the complete consumption of the starting material. The reaction mixture was concentrated under reduced pressure. The residue was taken up in ethyl acetate $(25 \mathrm{~mL})$ and washed with $10 \%$ aqueous $\mathrm{HCl}$ solution $(10 \mathrm{~mL})$ and then aqueous saturated sodium bicarbonate solution $(10 \mathrm{~mL})$. The organic layer was dried over magnesium sulfate and concentrated in vacuo. The crude product was purified by column chromatography using ethyl acetate/hexane 1:5 as the eluent to give the pure mesylate $6(1.06 \mathrm{~g}, 89 \%)$ as a colorless oil; $[\alpha]_{20}^{\mathrm{D}}=+59.9(\mathrm{c}=1.0$ in methanol $)$;

${ }^{1} \mathrm{H}$ NMR (600 MHz, DMSO- $d_{6}$, HSQC-DEPT, COSY, NOESY) $\delta$ 7.70-7.67 (m, 4H, Ph), 7.49-7.42 $(\mathrm{m}, 6 \mathrm{H}, \mathrm{Ph}), 5.28\left(\mathrm{~d}, J_{4,5}=9.9 \mathrm{~Hz}, 1 \mathrm{H}, \mathrm{H}-4\right), 4.21$ $\left(\mathrm{ddd} \approx \mathrm{dd}, J_{1 \mathrm{ax}, \text { leq }}=11.3 \mathrm{~Hz}, J_{\text {leq,2ax }}=7.7 \mathrm{~Hz}, J_{\text {leq,2eq }}\right.$ $<1 \mathrm{~Hz}, 1 \mathrm{H}, \mathrm{H}-1 \mathrm{eq}), 3.88\left(\mathrm{dd}, J_{5,6 \mathrm{a}}=1.8 \mathrm{~Hz}, 1 \mathrm{H}\right.$, H-6a), $3.85\left(\mathrm{dd}, J_{5,6 \mathrm{a}}=3.6 \mathrm{~Hz}, J_{6 \mathrm{a}, 6 \mathrm{~b}}=11.7 \mathrm{~Hz}, 1 \mathrm{H}\right.$, H-6b), 3.77 (ddd, 1H, H-5), 3.68 (ddd, $J_{1 \mathrm{ax}, 2 \mathrm{ax}}=13.5$ $\left.\mathrm{Hz}, J_{1 \mathrm{ax}, 2 \mathrm{eq}}=1.3 \mathrm{~Hz}, 1 \mathrm{H}, \mathrm{H}-1 \mathrm{ax}\right), 3.25(\mathrm{~s}, 3 \mathrm{H}$, $\left.\mathrm{SO}_{2} \mathrm{CH}_{3}\right), 2.88\left(\mathrm{ddd} \approx \mathrm{dt}, J_{2 \mathrm{ax}, 2 \mathrm{eq}}=13.5 \mathrm{~Hz}, 1 \mathrm{H}\right.$, H-2ax), 2.44 (ddd $\approx$ br. d, 1H, H-2eq), 1.00 (s, 9H, $t \mathrm{Bu}$;

${ }^{1} \mathrm{H}$ NMR (600 MHz, $\mathrm{CDCl}_{3}$, HSQC-DEPT, COSY, NOESY) $\delta$ 7.76-7.73 (m, 4H, Ph), 7.45-7.38 (m, 6H, $\mathrm{Ph}), 5.45\left(\mathrm{~d}, J_{4,5}=9.8 \mathrm{~Hz}, 1 \mathrm{H}, \mathrm{H}-4\right), 4.32(\mathrm{ddd} \approx \mathrm{dd}$, $J_{1 \mathrm{ax}, \text { leq }}=11.4 \mathrm{~Hz}, J_{\text {leq,2ax }}=7.5 \mathrm{~Hz}, J_{1 \mathrm{eq}, 2 \mathrm{eq}}=1.0 \mathrm{~Hz}$, $1 \mathrm{H}, \mathrm{H}-1 \mathrm{eq}), 3.97$ (dd, $J_{5,6 \mathrm{a}}=1.5 \mathrm{~Hz}, J_{6 \mathrm{a}, 6 \mathrm{~b}}=11.8 \mathrm{~Hz}$, $1 \mathrm{H}, \mathrm{H}-6 \mathrm{a}), 3.93\left(\mathrm{dd}, J_{5,6 \mathrm{a}}=3.0 \mathrm{~Hz}, J_{6 \mathrm{a}, 6 \mathrm{~b}}=11.8 \mathrm{~Hz}\right.$, $1 \mathrm{H}, \mathrm{H}-6 \mathrm{~b}), 3.64$ (ddd, $J_{1 \mathrm{ax}, 2 \mathrm{ax}}=12.6 \mathrm{~Hz}, J_{1 \mathrm{ax}, 2 \mathrm{eq}}=2.4$ $\mathrm{Hz}, 1 \mathrm{H}, \mathrm{H}-1 \mathrm{ax}$ ), 3.62 (ddd, 1H, H-5), 3.25 (s, 3H, $\mathrm{SO}_{2} \mathrm{CH}_{3}$ ), 2.82 (dddd $\approx \mathrm{ddt}, J_{\text {leq, } 2 \mathrm{ax}}=7.5 \mathrm{~Hz}$, $\left.J_{2 \mathrm{ax}, 2 \mathrm{eq}}=14.3 \mathrm{~Hz}, J_{2 \mathrm{ax}, 4}=0.8 \mathrm{~Hz}, 1 \mathrm{H}, \mathrm{H}-2 \mathrm{ax}\right), 2.58$ $\left(\mathrm{ddd}, J_{2 \mathrm{ax}, 2 \mathrm{eq}}=14.3 \mathrm{~Hz}, 1 \mathrm{H}, \mathrm{H}-2 \mathrm{eq}\right), 1.08(\mathrm{~s}, 9 \mathrm{H}$, $t \mathrm{Bu}$;
${ }^{13} \mathrm{C}$ NMR (151 MHz, DMSO- $\left.d_{6}\right) \delta 201.1(\mathrm{C}=\mathrm{O})$, $135.3(2 \mathrm{C}, o \mathrm{Ph}), 135.2(2 \mathrm{C}, o \mathrm{Ph}), 132.8$ (Si-CAr), 132.6 (Si-CAr), $130.0(p \mathrm{Ph}), 129.9(p \mathrm{Ph}), 127.92$ (2C, $m \mathrm{Ph}), 127.89$ (2C, $m \mathrm{Ph}), 80.1(\mathrm{C}-5), 78.5(\mathrm{C}-4)$, 66.0 (C-1), 62.7 (C-6), 41.9 (C-2), $38.8\left(\mathrm{SCH}_{3}\right), 26.6$ $(3 \mathrm{C}, t \mathrm{Bu}), 19.0(\mathrm{SiC}){ }^{13} \mathrm{C} \mathrm{NMR}\left(151 \mathrm{MHz}, \mathrm{CDCl}_{3}\right) \delta$ $201.6(\mathrm{C}=\mathrm{O}), 136.0(2 \mathrm{C}, o \mathrm{Ph}), 135.9(2 \mathrm{C}, o \mathrm{Ph})$, 133.14 (Si-CAr), 133.10 (Si-CAr), $129.9(p \mathrm{Ph})$, $129.8(p \mathrm{Ph}), 127.9(2 \mathrm{C}, m \mathrm{Ph}), 127.8(2 \mathrm{C}, m \mathrm{Ph}), 81.5$ (C-5), 78.9 (C-4), 66.8 (C-1), 62.5 (C-6), 42.4 (C-2), $39.8\left(\mathrm{SCH}_{3}\right), 26.9(3 \mathrm{C}, t \mathrm{Bu}), 19.5(\mathrm{SiC})$;

HRMS (mmi pos.) $\mathrm{m} / \mathrm{z} 462.1532$ calcd. for $\mathrm{C}_{23} \mathrm{H}_{30} \mathrm{O}_{6} \mathrm{SSi}$, found 462.1519 for $[\mathrm{M}]^{+}$.

\section{1,5-Anhydro-6-O-tert-butyldimethylsilyl-2-deoxy- D-erythro-hex-1-en-3-ulose (7)}

To a solution of compound $1(1.84 \mathrm{~g}, 12.80 \mathrm{mmol})$ in dichloromethane $(25 \mathrm{~mL})$ were added $t$-butyldimethylchlorosilane $(2.51 \mathrm{~g}, 16.64 \mathrm{mmol})$ and imidazole $(2.18 \mathrm{~g}, 32.01 \mathrm{mmol})$. The reaction mixture was stirred at room temperature for $30 \mathrm{~min}$. After this time TLC (ethyl acetate) showed the complete consumption of the starting material and the formation of one major product. The reaction mixture was washed with water $(3 \times 50 \mathrm{~mL})$, and the organic layer was dried over sodium sulfate and concentrated under reduced pressure. The resulting residue was purified by column chromatography (ethyl acetate/hexane 1:5) to give silylated compound $7(2.85 \mathrm{~g}, 86 \%)$ as a colorless crystals, mp. 113.8-114.7 ${ }^{\circ} \mathrm{C}$ (lit. 12: mp 114.4-115 ${ }^{\circ} \mathrm{C}$, lit. 13: $\left.114.5-115^{\circ} \mathrm{C}\right) ;[\alpha]_{20}^{\mathrm{D}}=+171.1(\mathrm{c}=0.5$ in methanol) (lit. 13: $[\alpha]_{20}^{\mathrm{D}}=+275.5 \quad(\mathrm{c}=0.25$ in chloroform);

${ }^{1} \mathrm{H}$ NMR (300 MHz, DMSO-d $\mathrm{d}_{6}, \mathrm{HSQC}, \mathrm{HMBC}$, NOESY) $\delta 7.61\left(\mathrm{~d}, J_{1,2}=5.8 \mathrm{~Hz}, 1 \mathrm{H}, \mathrm{H}-1\right), 5.68$ $\left(\mathrm{m}_{\mathrm{c}}, J_{1,2}=3.8 \mathrm{~Hz}, J_{4-\mathrm{OH}, 5}=1.3 \mathrm{~Hz}, 1 \mathrm{H}, 4-\mathrm{OH}\right), 5.30$ (d, 1H, H-2), 4.19-4.07 (m, 2H, H-4, H-5), 3.96 (dd, $\left.J_{5,6 \mathrm{a}}=1.4 \mathrm{~Hz}, J_{6 \mathrm{a}, 6 \mathrm{~b}}=11.8 \mathrm{~Hz}, 1 \mathrm{H}, \mathrm{H}-6 \mathrm{a}\right), 3.90(\mathrm{dd}$, $\left.J_{5,6 \mathrm{~b}}=3.2 \mathrm{~Hz}, J_{6 \mathrm{a}, 6 \mathrm{~b}}=11.8 \mathrm{~Hz}, 1 \mathrm{H}, \mathrm{H}-6 \mathrm{~b}\right), 0.86$ $(\mathrm{s}, 9 \mathrm{H}, t \mathrm{Bu}), 0.07\left(\mathrm{~s}, 6 \mathrm{H}, \mathrm{SiCH}_{3}\right) ;{ }^{1} \mathrm{H}$ NMR $(600$ $\left.\mathrm{MHz} \mathrm{CDCl}_{3}, \mathrm{HSQC}-\mathrm{DEPT}\right) \delta 7.41\left(\mathrm{~d}, J_{1,2}=5.8 \mathrm{~Hz}\right.$, $1 \mathrm{H}, \mathrm{H}-1), 5.45$ (d, $\left.J_{1,2}=5.8 \mathrm{~Hz}, 1 \mathrm{H}, \mathrm{H}-2\right), 4.35$ (d, $\left.J_{4,5}=13.3 \mathrm{~Hz}, 1 \mathrm{H}, \mathrm{H}-4\right), 4.12$ (dddd, ${ }^{4} J=0.6 \mathrm{~Hz}$, $1 \mathrm{H}, \mathrm{H}-5), 4.08\left(\mathrm{dd}, J_{5,6 \mathrm{a}}=1.9 \mathrm{~Hz}, J_{6 \mathrm{a}, 6 \mathrm{~b}}=11.9 \mathrm{~Hz}\right.$, $1 \mathrm{H}, \mathrm{H}-6 \mathrm{a}), 4.01\left(\mathrm{dd}, J_{5,6 \mathrm{~b}}=4.0 \mathrm{~Hz}, J_{6 \mathrm{a}, 6 \mathrm{~b}}=11.9 \mathrm{~Hz}\right.$, $1 \mathrm{H}, \mathrm{H}-6 \mathrm{~b}), 3.49$ (s, 1H, 4-OH), $0.92(\mathrm{~s}, 9 \mathrm{H}, t \mathrm{Bu})$, 0.12 (s, $\left.3 \mathrm{H}, \mathrm{CH}_{3}\right), 0.11$ (s, $\left.3 \mathrm{H}, \mathrm{CH}_{3}\right)$;

${ }^{13} \mathrm{C}$ NMR (75 MHz, DMSO-d 6$) \delta 194.3(\mathrm{C}=\mathrm{O})$, 163.5 (C-1), 103.8 (C-2), 83.0 (C-5), 67.3 (C-4), 61.7 (C-6), $25.8\left(3 \mathrm{C}, t \mathrm{Bu}-\mathrm{CH}_{3}\right), 18.1\left(\underline{\left.\mathrm{C}-\mathrm{CH}_{3}\right),-5.3}\right.$ $\left(2 \mathrm{C}, \mathrm{SiCH}_{3}\right)$;

${ }^{13} \mathrm{C}$ NMR $\left(151 \mathrm{MHz}, \mathrm{CDCl}_{3}\right) \delta 194.7(\mathrm{C}=\mathrm{O}), 164.6$ (C-1), 103.2 (C-2), 83.8 (C-5), 67.5 (C-4), 62.0 $(\mathrm{C}-6), 25.5\left(3 \mathrm{C}, t \mathrm{Bu}-\underline{C H}_{3}\right), 18.6\left(\underline{\mathrm{C}}-\mathrm{CH}_{3}\right),-5.36$, $5.39\left(2 \mathrm{C}, \mathrm{Si}-\mathrm{CH}_{3}\right)$.

\section{1,5-Anhydro-6-O-tert-butyldimethylsilyl-2-deoxy- 4-O-methanesulphonyl-D-erythro-hex-1-en-3- ulose (8)}

To a solution of compound $7(2.55 \mathrm{~g}, 9.87 \mathrm{mmol})$ in dry pyridine $(12 \mathrm{~mL})$ was added methanesulfonyl 
chloride $(0.84 \mathrm{~mL}, 10.86 \mathrm{mmol})$ at $0{ }^{\circ} \mathrm{C}$. The reaction mixture was stirred for $4 \mathrm{~h}$ at $\mathrm{rt}$. After this time TLC (AcOEt/hexane 1:3) indicated the complete consumption of the starting material. The reaction mixture was concentrated under reduced pressure. The residue was taken up in ethyl acetate $(25 \mathrm{~mL})$ and washed with $10 \%$ aqueous $\mathrm{HCl}$ solution $(20 \mathrm{~mL})$ and then aqueous saturated sodium bicarbonate solution $(20 \mathrm{~mL})$. The organic layer was dried over sodium sulfate and concentrated in vacuo. The crude product was purified by column chromatography using ethyl acetate/hexane $1: 5$ as the eluent to give the pure mesylate $8(2.55 \mathrm{~g}, 77 \%)$ as a colorless oil; $[\alpha]_{20}^{\mathrm{D}}=+224.0 \quad(\mathrm{c}=0.1$ in methanol);

${ }^{1} \mathrm{H}$ NMR (300 MHz, DMSO-d 6 , HSQC, HMBC, COSY) $\delta 7.74\left(\mathrm{~d}, J_{1,2}=5.9 \mathrm{~Hz}, 1 \mathrm{H}, \mathrm{H}-1\right), 5.46$ $\left(\mathrm{d}, J_{1,2}=5.8 \mathrm{~Hz}, 1 \mathrm{H}, \mathrm{H}-2\right), 5.28\left(\mathrm{~d}, J_{4,5}=11.8 \mathrm{~Hz}\right.$, $1 \mathrm{H}, \mathrm{H}-4), 4.64$ (ddd $\left.\approx \mathrm{dt}, 1 \mathrm{H}, J_{4,5}=11.7 \mathrm{~Hz}, \mathrm{H}-5\right)$, $3.98\left(\mathrm{dd}, J_{5,6 a}=2.0 \mathrm{~Hz}, J_{6 \mathrm{a}, 6 \mathrm{~b}}=12.4 \mathrm{~Hz}, 1 \mathrm{H}, \mathrm{H}-6 \mathrm{a}\right)$, $3.90\left(\mathrm{dd}, J_{5,6 \mathrm{~b}}=3.3 \mathrm{~Hz}, J_{6 \mathrm{a}, 6 \mathrm{~b}}=12.4 \mathrm{~Hz}, 1 \mathrm{H}, \mathrm{H}-6 \mathrm{~b}\right)$, $3.34\left(\mathrm{~s}, 3 \mathrm{H}, \mathrm{SCH}_{3}\right), 0.87(\mathrm{~s}, 6 \mathrm{H}, t \mathrm{Bu}), 0.08(\mathrm{~s}, 3 \mathrm{H}$, $t \mathrm{Bu}), 0.07$ (s, 3H, $\left.\mathrm{SiCH}_{3}\right), 0.07$ (s, 3H, $\mathrm{SiCH}_{3}$ );

${ }^{1} \mathrm{H}$ NMR (600 MHz, DMSO-d 6 , HSQC-DEPT) $\delta 7.39\left(\mathrm{~d}, J_{1,2}=5.9 \mathrm{~Hz}, 1 \mathrm{H}, \mathrm{H}-1\right), 5.45\left(\mathrm{~d}, J_{1,2}=5.9\right.$ $\mathrm{Hz}, 1 \mathrm{H}, \mathrm{H}-2), 5.43$ (d, $\left.J_{4,5}=12.1 \mathrm{~Hz}, 1 \mathrm{H}, \mathrm{H}-4\right), 4.43$ $\left(\mathrm{ddd} \approx \mathrm{dt}, J_{4,5}=12.1 \mathrm{~Hz}, 1 \mathrm{H}, \mathrm{H}-5\right), 4.04(\mathrm{dd}$, $\left.J_{5,6 a}=2.0 \mathrm{~Hz}, J_{6 \mathrm{a}, 6 \mathrm{~b}}=12.0 \mathrm{~Hz}, 1 \mathrm{H}, \mathrm{H}-6 \mathrm{a}\right), 3.99(\mathrm{dd}$, $\left.J_{5,6 \mathrm{~b}}=3.2 \mathrm{~Hz}, J_{6 \mathrm{a}, 6 \mathrm{~b}}=12.0 \mathrm{~Hz}, 1 \mathrm{H}, \mathrm{H}-6 \mathrm{~b}\right), 3.33$ (s, 3H, $\left.\mathrm{SCH}_{3}\right), 0.92(\mathrm{~s}, 9 \mathrm{H}, t \mathrm{Bu}), 0.12\left(\mathrm{~s}, 3 \mathrm{H}, \mathrm{SiCH}_{3}\right)$, $0.11\left(\mathrm{~s}, 3 \mathrm{H}, \mathrm{SiCH}_{3}\right) ;{ }^{13} \mathrm{C}$ NMR $\left(75 \mathrm{MHz}, \mathrm{DMSO}-\mathrm{d}_{6}\right)$ $\delta 187.3(\mathrm{C}=\mathrm{O}), 164.5$ (C-1), 104.1 (C-2), 80.1 (C-5), 73.8 (C-4), 60.9 (C-6), $38.7\left(\mathrm{SCH}_{3}\right), 25.7$ (tBu), 18.0 ( $\mathrm{SiC}),-5.4\left(\mathrm{SiCH}_{3}\right)$;

${ }^{13} \mathrm{C}$ NMR (151 MHz, DMSO-d 6$) \delta 188.4(\mathrm{C}=\mathrm{O})$, 163.6 (C-1), 104.8 (C-2), 81.3 (C-5), 74.4 (C-4), $61.1(\mathrm{C}-6), 39.8\left(\mathrm{SCH}_{3}\right), 25.9(t \mathrm{Bu}), 18.5(\mathrm{SiC}),-5.4$ $\left(\mathrm{SiCH}_{3}\right)$; HRMS (GC TOF, EI) $\mathrm{m} / z 321.08226$ calcd. for $\mathrm{C}_{12} \mathrm{H}_{21} \mathrm{O}_{6} \mathrm{SSi}$, found 321.08073 for $\left[\mathrm{M}-\mathrm{CH}_{3}\right]^{+}$; $m / z$ 2 279.03706 calcd. for $\mathrm{C}_{9} \mathrm{H}_{15} \mathrm{O}_{6} \mathrm{SSi}$, found 279.03415 for $\left[\mathrm{M}-t \mathrm{Bu}^{\cdot}\right]^{+}$.

1,5-Anhydro-6-O-tert-butyldimethylsilyl-2-deoxyD-erythro-hex-3-ulose (9) and 1,5-Anhydro-6-O tert-butyldimethylsilyl-2-deoxy-D-arabino-hexitol (10)

Through a solution of compound 7 (3.76 g, 14.68 $\mathrm{mmol})$ in ethanol $(150 \mathrm{~mL})$ was bubbled argon for five minutes. Palladium-on-carbon $(10 \%, 188 \mathrm{mg})$ was added carefully. The reaction flask was evacuated, and hydrogen was added. The mixture was stirred for 20 minutes when the full conversion was detected (ethyl acetate/hexane 3:7). The catalyst was filtered off through celite, and the solvent was reduced in vacuo. The resulting crude oil was subjected to column chromatography (ethyl acetate /hexane 1:5) to furnish compounds $9(0.701 \mathrm{~g}, 18 \%)$ and $10(2.2347 \mathrm{~g}, 58 \%)$ both as colorless solids.

Compound 9:

Amorphous; $[\alpha]_{20}^{\mathrm{D}}=+30(\mathrm{c}=1.0$ in methanol);
${ }^{1} \mathrm{H}$ NMR (300 MHz, DMSO-d ${ }_{6}$, HSQC, HMBC, NOESY) $\delta 5.26\left(\mathrm{~d}, J_{4,4-\mathrm{OH}}=5.7 \mathrm{~Hz}, 1 \mathrm{H}, 4-\mathrm{OH}\right), 4.15$ $\left(\mathrm{ddd}, J_{1 \mathrm{ax}, 1 \text { eq }}=11.2 \mathrm{~Hz}, J_{1 \text { eq, } 2 \text { eq }}=1.1 \mathrm{~Hz}, J_{1 \text { eq, } 2 \mathrm{ax}}=7.4\right.$ $\mathrm{Hz}, 1 \mathrm{H}, \mathrm{H}-1 \mathrm{eq}$ ), 3.97 (ddd, ${ }^{4} J_{2 \mathrm{ax}, 4}=1.3 \mathrm{~Hz}, J_{4,4-\mathrm{OH}}=$ $\left.5.7 \mathrm{~Hz}, J_{4,5}=9.9 \mathrm{~Hz}, 1 \mathrm{H}, \mathrm{H}-4\right), 3.87\left(\mathrm{dd}, J_{5,6 \mathrm{a}}=1.7\right.$ $\left.\mathrm{Hz}, J_{6 \mathrm{a}, 6 \mathrm{~b}}=11.4 \mathrm{~Hz}, 1 \mathrm{H}, \mathrm{H}-6 \mathrm{a}\right), 3.76\left(\mathrm{dd}, J_{5,6 \mathrm{~b}}=4.9\right.$ $\left.\mathrm{Hz}, J_{6 \mathrm{a}, 6 \mathrm{~b}}=11.4 \mathrm{~Hz}, 1 \mathrm{H}, \mathrm{H}-6 \mathrm{~b}\right), 3.53$ (ddd, $J_{1 \mathrm{ax}, 1 \mathrm{eq}}=$ $11.3 \mathrm{~Hz}, J_{1 \mathrm{ax}, 2 \mathrm{eq}}=2.5 \mathrm{~Hz}, J_{1 \mathrm{ax}, 2 \mathrm{ax}}=12.6 \mathrm{~Hz}, 1 \mathrm{H}$, H-1ax), 3.28 (ddd, $J_{4,5}=9.9 \mathrm{~Hz}, J_{5,6 \mathrm{~b}}=4.9 \mathrm{~Hz}, 1 \mathrm{H}$, $\mathrm{H}-5), 2.70$ (dddd, $J_{1 \mathrm{ax}, 2 \mathrm{ax}}=12.6 \mathrm{~Hz}, J_{\text {leq, } 2 \mathrm{ax}}=7.4 \mathrm{~Hz}$, $\left.J_{2 \mathrm{ax}, 2 \mathrm{eq}}=14.0 \mathrm{~Hz},{ }^{4} J_{2 \mathrm{ax}, 4}=1.3 \mathrm{~Hz}, 1 \mathrm{H}, \mathrm{H}-2 \mathrm{ax}\right), 2.27$ $\left(\right.$ ddd, $\left.J_{1 \mathrm{eq}, 2 \mathrm{eq}}=1.1 \mathrm{~Hz}, J_{2 \mathrm{ax}, 2 \mathrm{eq}}=14.0 \mathrm{~Hz}, 1 \mathrm{H}, \mathrm{H}-2 \mathrm{eq}\right)$, $0.87(\mathrm{~s}, 9 \mathrm{H}, t \mathrm{Bu}), 0.05$ (s, 6H, $\left.\mathrm{SiCH}_{3}\right)$;

${ }^{1} \mathrm{H}$ NMR (600 MHz, $\mathrm{CDCl}_{3}$, HSQC-DEPT) $\delta 4.33$ $\left(\mathrm{ddd}, J_{1 \mathrm{ax}, \text { leq }}=11.3 \mathrm{~Hz}, J_{1 \mathrm{eq}, 2 \mathrm{eq}}=1.1 \mathrm{~Hz}, J_{\text {leq,2ax }}=7.6\right.$, $\mathrm{Hz}, 1 \mathrm{H}, \mathrm{H}-1 \mathrm{eq}$ ), 4.20 (ddd, $J_{4,5}=9.8 \mathrm{~Hz}, 1 \mathrm{H}, \mathrm{H}-4$ ), $3.99\left(\mathrm{dd}, J_{5,6 \mathrm{a}}=1.9 \mathrm{~Hz}, J_{6 \mathrm{a}, 6 \mathrm{~b}}=11.5 \mathrm{~Hz}, 1 \mathrm{H}, \mathrm{H}-6 \mathrm{a}\right)$, $3.90\left(\mathrm{dd}, J_{5,6 \mathrm{~b}}=4.4 \mathrm{~Hz}, J_{6 \mathrm{a}, 6 \mathrm{~b}}=11.5 \mathrm{~Hz}, 1 \mathrm{H}, \mathrm{H}-6 \mathrm{~b}\right)$, $3.63\left(\mathrm{ddd}, J_{1 \mathrm{ax}, 1 \mathrm{eq}}=11.3 \mathrm{~Hz}, J_{1 \mathrm{ax}, 2 \mathrm{eq}}=2.5 \mathrm{~Hz}, J_{1 \mathrm{ax}, 2 \mathrm{ax}}=\right.$ $12.5 \mathrm{~Hz}, 1 \mathrm{H}, \mathrm{H}-1 \mathrm{ax}), 3.56\left(\mathrm{~d}, J_{4,4-\mathrm{OH}}=3.7 \mathrm{~Hz}, 1 \mathrm{H}\right.$, 4- $\mathrm{OH}), 3.28$ (ddd, $\left.J_{4,5}=9.8 \mathrm{~Hz}, 1 \mathrm{H}, \mathrm{H}-5\right), 2.78$ (dddd, $J_{1 \mathrm{ax}, 2 \mathrm{ax}}=12.5 \mathrm{~Hz}, J_{1 \mathrm{eq}, 2 \mathrm{ax}}=7.6 \mathrm{~Hz}, J_{2 \mathrm{ax}, 2 \mathrm{eq}}=$ $13.9 \mathrm{~Hz},{ }^{4} J_{2 \mathrm{ax}, 4}=1.5 \mathrm{~Hz}, 1 \mathrm{H}, \mathrm{H}-2 \mathrm{ax}$ ), 2.53 (ddd, $\left.J_{1 \text { eq,2eq }}=1.1 \mathrm{~Hz}, J_{2 \mathrm{ax}, 2 \mathrm{eq}}=13.9 \mathrm{~Hz}, 1 \mathrm{H}, \mathrm{H}-2 \mathrm{eq}\right), 0.91$ $(\mathrm{s}, 9 \mathrm{H}, t \mathrm{Bu}), 0.11\left(\mathrm{~s}, 3 \mathrm{H}, \mathrm{CH}_{3}\right), 0.10\left(\mathrm{~s}, 3 \mathrm{H}, \mathrm{CH}_{3}\right)$;

${ }^{13} \mathrm{C}$ NMR (75 MHz, DMSO-d $\left.{ }_{6}\right) \delta 207.5(\mathrm{C}=\mathrm{O}), 83.6$ (C-5), 73.3 (C-4), 66.2 (C-1), 63.3 (C-6), 41.3 (C-2), $25.9\left(\mathrm{C}-\underline{C H}_{3}\right), 18.2\left(\underline{\mathrm{C}}-\mathrm{CH}_{3}\right),-5.16\left(\mathrm{Si}-\mathrm{CH}_{3}\right),-5.19$ $\left(\mathrm{SiCH}_{3}\right)$;

${ }^{13} \mathrm{C}$ NMR $\left(151 \mathrm{MHz}, \mathrm{CDCl}_{3}\right) \delta 208.2(\mathrm{C}=\mathrm{O}), 84.8$ (C-5), 73.9 (C-4), 67.5 (C-1), 63.5 (C-6), 41.0 (C-2), $26.1\left(3 \mathrm{C}, \mathrm{C}-\mathrm{CH}_{3}\right), 18.7\left(\underline{\mathrm{C}}-\mathrm{CH}_{3}\right),-5.1\left(2 \mathrm{C}, \mathrm{Si}-\mathrm{CH}_{3}\right)$; MS (IS pos.) $m / z 245.1\left[\mathrm{M}_{-} \mathrm{CH}_{3}\right]^{+}, 203.1[\mathrm{M}-t \mathrm{Bu}]^{+}$; HRMS (GC TOF) $\mathrm{m} / \mathrm{z} 245.12036$ calcd. for $\mathrm{C}_{11} \mathrm{H}_{21} \mathrm{O}_{4} \mathrm{Si}$, found 245.11812 for $\left[\mathrm{M}-\mathrm{CH}_{3}\right]^{+}, \mathrm{m} / \mathrm{z}$ 203.07341 calcd. for $\mathrm{C}_{8} \mathrm{H}_{15} \mathrm{O}_{4} \mathrm{Si}$, found 203.107168 for $[\mathrm{M}-t \mathrm{Bu}]^{+}$.

\section{Compound 10:}

Amorphous; $[\alpha]_{20}^{\mathrm{D}}=+11.4(\mathrm{c}=0.1$ in methanol);

${ }^{1} \mathrm{H}$ NMR (300 MHz, DMSO-d 6 , HSQC, HMBC, NOESY) $\delta 4.83\left(\mathrm{~d}, J_{4,4-\mathrm{OH}}=4.7 \mathrm{~Hz}, 1 \mathrm{H}, 4-\mathrm{OH}\right), 4.79$ $\left(\mathrm{d}, J_{3,3-\mathrm{OH}}=4.6 \mathrm{~Hz}, 1 \mathrm{H}, 3-\mathrm{OH}\right), 3.85\left(\mathrm{dd}, J_{5,6 \mathrm{a}}=1.6\right.$ $\left.\mathrm{Hz}, J_{6 \mathrm{a}, 6 \mathrm{~b}}=11.2 \mathrm{~Hz}, 1 \mathrm{H}, \mathrm{H}-6 \mathrm{a}\right), 3.76\left(\mathrm{ddd}, J_{1 \mathrm{ax}, 1 \mathrm{eq}}=\right.$ $11.5 \mathrm{~Hz}, J_{\text {leq,2ax }}=4.8 \mathrm{~Hz}, J_{\text {leq,2eq }}=1.6 \mathrm{~Hz}, 1 \mathrm{H}$, $\mathrm{H}-1 \mathrm{eq}), 3.62\left(\mathrm{dd}, J_{5,6 \mathrm{~b}}=5.4 \mathrm{~Hz}, J_{6 \mathrm{a}, 6 \mathrm{~b}}=11.2 \mathrm{~Hz}, 1 \mathrm{H}\right.$, $\mathrm{H}-6 \mathrm{~b}), 3.36-3.26(\mathrm{~m}, 1 \mathrm{H}, \mathrm{H}-3), 3.26$ (ddd, $J_{1 \mathrm{ax}, \text { leq }}=$ $11.5 \mathrm{~Hz}, J_{1 \mathrm{ax}, 2 \mathrm{eq}}=2.0 \mathrm{~Hz}, J_{1 \mathrm{ax}, 2 \mathrm{ax}}=12.6 \mathrm{~Hz}, 1 \mathrm{H}$, $\mathrm{H}-1 \mathrm{ax}), 2.96$ (ddd, $1 \mathrm{H}, J_{5,6 \mathrm{a}}=1.8 \mathrm{~Hz}, J_{5,6 \mathrm{~b}}=5.5 \mathrm{~Hz}$, $\mathrm{H}-5), 2.92\left(\mathrm{ddd} \approx \mathrm{dt}, 1 \mathrm{H}, J_{4,4-\mathrm{OH}}=4.7 \mathrm{~Hz}, \mathrm{H}-4\right), 1.74$ $\left(\mathrm{dddd} \approx \mathrm{ddt}, J_{2 \mathrm{ax}, 2 \mathrm{eq}}=12.9 \mathrm{~Hz}, J_{2 \mathrm{eq}, 3}=5.0 \mathrm{~Hz}, 1 \mathrm{H}\right.$, H-2eq), 1.38 (dddd $\approx$ ddt, $J_{1 \text { ax }, 2 \mathrm{ax}}=12.6 \mathrm{~Hz}, J_{1 \mathrm{eq}, 2 \mathrm{ax}}=$ $4.8 \mathrm{~Hz}, J_{2 \mathrm{ax}, 2 \mathrm{eq}}=12.8 \mathrm{~Hz}, J_{2 \mathrm{ax}, 3}=11.5 \mathrm{~Hz}, 1 \mathrm{H}$, $\mathrm{H}-2 \mathrm{ax}), 0.86(\mathrm{~s}, 9 \mathrm{H}, t \mathrm{Bu}), 0.03\left(\mathrm{~s}, 3 \mathrm{H}, \mathrm{SiCH}_{3}\right), 0.02$ (s, 3H, $\mathrm{SiCH}_{3}$ );

${ }^{1} \mathrm{H}$ NMR (600 MHz, $\mathrm{CDCl}_{3}$, HSQC-DEPT) $\delta 3.92$ $\left(\mathrm{ddd}, J_{1 \mathrm{ax}, \text { leq }}=11.7 \mathrm{~Hz}, J_{\text {leq,2eq }}=1.6 \mathrm{~Hz}, J_{1 \text { eq, } 2 \mathrm{ax}}=4.9\right.$ $\mathrm{Hz}, 1 \mathrm{H}, \mathrm{H}-1 \mathrm{eq}), 3.91$ (dd, $J_{5,6 \mathrm{a}}=4.6 \mathrm{~Hz}, J_{6 \mathrm{a}, 6 \mathrm{~b}}=10.2$ $\mathrm{Hz}, 1 \mathrm{H}, \mathrm{H}-6 \mathrm{a}), 3.74\left(\mathrm{dd}, J_{5,6 \mathrm{~b}}=7.0 \mathrm{~Hz}, J_{6 \mathrm{a}, 6 \mathrm{~b}}=10.2\right.$ $\mathrm{Hz}, 1 \mathrm{H}, \mathrm{H}-6 \mathrm{~b}$ ), 3.65 (ddd, $J_{2 \mathrm{ax}, 3}=11.5 \mathrm{~Hz}, J_{2 \mathrm{eq}, 3}=5.0$ $\left.\mathrm{Hz}, J_{3,4}=8.7 \mathrm{~Hz}, 1 \mathrm{H}, \mathrm{H}-3\right), 3.44\left(\mathrm{ddd}, J_{1 \mathrm{ax}, 1 \mathrm{eq}}=11.7\right.$ 
$\left.\mathrm{Hz}, J_{1 \mathrm{ax}, 2 \mathrm{eq}}=2.0 \mathrm{~Hz}, J_{1 \mathrm{ax}, 2 \mathrm{ax}}=12.7 \mathrm{~Hz}, 1 \mathrm{H}, \mathrm{H}-1 \mathrm{ax}\right)$, $3.19\left(\mathrm{ddd}, J_{4,5}=9.1 \mathrm{~Hz}, J_{5,6 \mathrm{a}}=4.6 \mathrm{~Hz}, J_{5,6 \mathrm{~b}}=7.0 \mathrm{~Hz}\right.$, $1 \mathrm{H}, \mathrm{H}-5), 3.41(\mathrm{dd} \approx \mathrm{t}, 1 \mathrm{H}, \mathrm{H}-4), 1.91$ (dddd $\approx \mathrm{ddt}$, $J_{1 \mathrm{ax}, 2 \mathrm{eq}}=2.1 \mathrm{~Hz}, J_{2 \mathrm{ax}, 2 \mathrm{eq}}=13.1 \mathrm{~Hz}, J_{2 \mathrm{eq}, 3}=5.0 \mathrm{~Hz}, 1 \mathrm{H}$, $\mathrm{H}-2 \mathrm{eq}$ ), 1.66 (dddd $\approx \mathrm{ddt}, J_{1 \mathrm{ax}, 2 \mathrm{ax}}+J_{2 \mathrm{ax}, 2 \mathrm{eq}}+J_{2 \mathrm{ax}, 3}=$ $\left.37.2 \mathrm{~Hz}, J_{\text {leq, } 2 \mathrm{ax}}=4.9 \mathrm{~Hz}, 1 \mathrm{H}, \mathrm{H}-2 \mathrm{ax}\right), 0.90(\mathrm{~s}, 9 \mathrm{H}$, $t \mathrm{Bu}), 0.10\left(\mathrm{~s}, 3 \mathrm{H}, \mathrm{CH}_{3}\right), 0.09$ (s, 3H, $\left.\mathrm{CH}_{3}\right)$;

${ }^{13} \mathrm{C}$ NMR (75 MHz, DMSO-d 6 ) $\delta 81.4(\mathrm{C}-5), 72.3$ (C-3), 71.7 (C-4), 64.8 (C-1), 63.5 (C-6), 34.1 (C-2), $25.9(t \mathrm{Bu}), 18.2(\mathrm{SiC}),-5.11\left(\mathrm{SiCH}_{3}\right),-5.12\left(\mathrm{SiCH}_{3}\right)$;

${ }^{13} \mathrm{C}$ NMR $\left(151 \mathrm{MHz}, \mathrm{CDCl}_{3}\right) \delta 77.7(\mathrm{C}-4), 76.4$ (C-5), 73.1 (C-3), 65.9 (C-1), 65.8 (C-6), 32.8 (C-2), $26.0\left(3 \mathrm{C}, t \mathrm{Bu}-\underline{\mathrm{CH}}_{3}\right), 18.4\left(\underline{\mathrm{C}}-\mathrm{CH}_{3}\right),-5.38\left(\mathrm{Si}-\mathrm{CH}_{3}\right)$, $5.40\left(\mathrm{Si}-\mathrm{CH}_{3}\right)$;

$\mathrm{MS}\left(\mathrm{CI}, \mathrm{NH}_{3}\right) \mathrm{m} / z$ 205.1 [M- $\left.t \mathrm{Bu}^{-}\right]^{+}$

HRMS (GC TOF) $\mathrm{m} / z \quad 205.08906$ calcd. for $\mathrm{C}_{8} \mathrm{H}_{17} \mathrm{O}_{4} \mathrm{Si}$, found 205.08961 for [M- $\left.t \mathrm{Bu}\right]^{+}$.

1,5-Anhydro-6-O-tert-butyldiphenylsilyl-2-deoxy4-O-methanesulphonyl-D-arabino-hexitol (11), 1,5-Anhydro-6-O-tert-butyldiphenylsilyl-2-deoxy4-O-methanesulphonyl-D-ribo-hexitol (12), and 2,6;3,4-Bis-anhydro-6-O-tert-butyldiphenylsilyl-5deoxy-D-arabino-hexitol (13)

To a solution of compound $6(1.68 \mathrm{~g}, 3.62 \mathrm{mmol})$ in a 5:1 mixture of ethanol and water $(30 \mathrm{~mL})$ was added a solution of sodium borohydride $(0.179 \mathrm{~g}$, $4.72 \mathrm{mmol})$ in water $(3 \mathrm{~mL})$. The reaction mixture was then stirred for $10 \mathrm{~min}$ at room temperature. After this time TLC (ethyl acetate/hexane 1:1) showed the complete consumption of the starting material. The reaction mixture was diluted with ethyl acetate $(30 \mathrm{~mL})$, and the aqueous layer was separated and extracted with ethyl acetate $(2 \times 30 \mathrm{~mL})$, dried over sodium sulfate, and concentrated in vacuo. The crude product was purified by flash column chromatography (ethyl acetate/hexane 1:3) to give compounds 11 and $12(1.48 \mathrm{~g}, 88 \%, 11 / 12=83: 17)$ along with epoxide $13(0.12 \mathrm{~g}, 9 \%)$.

Compound 11:

Amorphous; $[\alpha]^{\mathrm{D}}{ }_{20}=+20.0(\mathrm{c}=0.1$ in methanol);

${ }^{1} \mathrm{H}$ NMR (600 MHz, $\mathrm{CDCl}_{3}$, HSQC-DEPT) $\delta 7.71-$ $7.70(\mathrm{~m}, 4 \mathrm{H}, \mathrm{Ph})$ 7.44-7.40 (m, 2H, Ph), 7.39-7.36 $(\mathrm{m}, 4 \mathrm{H}, \mathrm{Ph}), 4.59\left(\mathrm{dd} \approx \mathrm{t}, J_{3,4}=8.8 \mathrm{~Hz}, 1 \mathrm{H}, \mathrm{H}-4\right)$, $4.00\left(\mathrm{ddd}, J_{1 \mathrm{ax}, \text { leq }}=11.8 \mathrm{~Hz}, J_{1 \mathrm{eq}, 2 \mathrm{ax}}=4.9 \mathrm{~Hz}, J_{\text {leq,2eq }}=\right.$ $1.6 \mathrm{~Hz}, 1 \mathrm{H}, \mathrm{H}-1 \mathrm{eq}), 3.90\left(\mathrm{dd}, J_{5,6 \mathrm{a}}=1.8 \mathrm{~Hz}, J_{6 \mathrm{a}, 6 \mathrm{~b}}=\right.$ $11.7 \mathrm{~Hz}, 1 \mathrm{H}, \mathrm{H}-6 \mathrm{a}), 3.84\left(\mathrm{ddd} \approx \mathrm{dt}, J_{2 \mathrm{eq}, 3}=5.3 \mathrm{~Hz}\right.$, $1 \mathrm{H}, \mathrm{H}-3), 3.83\left(\mathrm{dd}, J_{5,6 \mathrm{~b}}=4.2 \mathrm{~Hz}, J_{6 \mathrm{a}, 6 \mathrm{~b}}=11.7 \mathrm{~Hz}\right.$, $1 \mathrm{H}, \mathrm{H}-6 \mathrm{~b}), 3.39$ (ddd $\approx \mathrm{dt}, J_{1 \mathrm{ax}, 1 \mathrm{eq}}=11.8 \mathrm{~Hz}, J_{1 \mathrm{ax}, 2 \mathrm{ax}}=$ $\left.13.7 \mathrm{~Hz}, J_{1 \mathrm{ax}, 2 \mathrm{eq}}=1.9 \mathrm{~Hz}, 1 \mathrm{H}, \mathrm{H}-1 \mathrm{ax}\right), 3.30$ (ddd, $J_{5,6 \mathrm{a}}$ $\left.=1.8 \mathrm{~Hz}, J_{5,6 \mathrm{~b}}=4.2 \mathrm{~Hz}, 1 \mathrm{H}, \mathrm{H}-5\right), 2.96\left(\mathrm{~s}, 3 \mathrm{H}, \mathrm{CH}_{3}\right)$, 2.09 (dddd $\approx \mathrm{ddt}, J_{2 \mathrm{ax}, 2 \mathrm{eq}}=13.3 \mathrm{~Hz}, J_{2 \mathrm{eq}, 3}=5.3 \mathrm{~Hz}$, $1 \mathrm{H}, \mathrm{H}-2 \mathrm{eq}$ ), 1.76 (dddd, $J_{2 \mathrm{ax}, 3}=10.5 \mathrm{~Hz}, 1 \mathrm{H}, \mathrm{H}-2 \mathrm{ax}$ ), $1.07(\mathrm{~s}, 9 \mathrm{H}, \mathrm{tBu})$;

${ }^{1} \mathrm{H}$ NMR (300 MHz, DMSO- $d_{6}$, HSQC, HMBC, NOESY) $\delta$ 7.71-7.62 (m, 4H, oPh), 7.47-7.36 $(\mathrm{m}, 6 \mathrm{H}, m \mathrm{Ph}, p \mathrm{Ph}), 5.51\left(\mathrm{~d}, J_{3,3-\mathrm{OH}}=6.2 \mathrm{~Hz}, 1 \mathrm{H}\right.$, $3-\mathrm{OH}), 4.38\left(\mathrm{dd} \approx \mathrm{t}, J_{3,4}+J_{4,5}=18.4 \mathrm{~Hz}, 1 \mathrm{H}, \mathrm{H}-4\right)$, $3.86\left(\mathrm{ddd}, J_{1 \text { ax }, \text { leq }}=11.7 \mathrm{~Hz}, J_{\text {leq,2ax }}=5.0 \mathrm{~Hz}, J_{\text {leq,2eq }}=\right.$ $1.5 \mathrm{~Hz}, \mathrm{H}-1 \mathrm{eq}$ ), 3.69 (ddd $\approx \mathrm{dt}, J_{2 \mathrm{eq}, 3}=5.3 \mathrm{~Hz}, 1 \mathrm{H}$, H-3), $3.86\left(\mathrm{dd}, J_{5,6 \mathrm{a}}=1.8 \mathrm{~Hz}, J_{6 \mathrm{a}, 6 \mathrm{~b}}=11.5 \mathrm{~Hz}, 1 \mathrm{H}\right.$,
H-6a), $3.78\left(\mathrm{dd}, J_{5,6 \mathrm{~b}}=4.5 \mathrm{~Hz}, J_{6 \mathrm{a}, 6 \mathrm{~b}}=11.5 \mathrm{~Hz}, 1 \mathrm{H}\right.$, 6b), 3.42-3.29 (m, 2H, H-1ax, H-5), 3.23 (s, 3H, $\mathrm{SCH}_{3}$ ), 1.92 (dddd $\approx$ br. dd, $J_{2 \mathrm{ax}, 2 \mathrm{eq}}=13.3 \mathrm{~Hz}, 1 \mathrm{H}$, $\mathrm{H}-2 \mathrm{eq}), 1.58$ (dddd $\approx \mathrm{dq}, J_{1 \mathrm{ax}, 2 \mathrm{ax}}+J_{2 \mathrm{ax}, 3}=23.6 \mathrm{~Hz}$, $\left.J_{\text {leq,2ax }}=4.9 \mathrm{~Hz}, 1 \mathrm{H}, \mathrm{H}-2 \mathrm{ax}\right), 0.98(\mathrm{~s}, 9 \mathrm{H}, t \mathrm{Bu})$;

${ }^{13} \mathrm{C} \mathrm{NMR}\left(75 \mathrm{MHz}, \mathrm{CDCl}_{3}\right) \delta 136.0(2 \mathrm{C}, o \mathrm{Ph}), 135.8$ (2C,oPh), 133.6 (Si-CAr), 133.3 (Si-CAr), 129.9 $(p \mathrm{Ph}), \quad 129.8 \quad(p \mathrm{Ph}), \quad 127.8 \quad(2 \mathrm{C}, \quad m \mathrm{Ph}), \quad 127.7$ (2C, $m \mathrm{Ph}), 81.0(\mathrm{C}-4), 78.5(\mathrm{C}-5), 71.4(\mathrm{C}-3), 65.4$ (C-1), 63.0 (C-6), $38.3\left(\mathrm{SO}_{2} \mathrm{CH}_{3}\right), 34.0$ (C-2), 27.0 $\left(3 \mathrm{C}, \mathrm{CH}_{3}\right), 19.5(\mathrm{SiC})$;

${ }^{13} \mathrm{C}$ NMR (75 MHz, DMSO- $\left.d_{6}\right) \delta 135.3(2 \mathrm{C}, o \mathrm{Ph})$, $135.2(2 \mathrm{C}, o \mathrm{Ph}), 133.1$ (Si-CPh), $133.0(\mathrm{Si}-\mathrm{CPh})$, $129.7(2 \mathrm{C}, p \mathrm{Ph}), 128.0(4 \mathrm{C}, m \mathrm{Ph}), 81.4(\mathrm{C}-4), 78.2$ (C-5), 69.8 (C-3), 64.7 (C-1), 62.8 (C-6), 38.7 $\left(\mathrm{SO}_{2} \mathrm{CH}_{3}\right), 34.7(\mathrm{C}-2), 26.6(3 \mathrm{C}, t \mathrm{Bu}), 18.9(\mathrm{SiC})$;

HRMS (nanospray in $\mathrm{NH}_{4} \mathrm{Ac} / \mathrm{CH}_{3} \mathrm{CN}$ ) $\mathrm{m} / \mathrm{z}$ 482.20736 calcd. for $\mathrm{C}_{23} \mathrm{H}_{36} \mathrm{O}_{6} \mathrm{NSSi}$, found 482.20295 for $\left[\mathrm{M}+\mathrm{NH}_{4}{ }^{+}\right]^{+}$;

HRMS (mmi neg.) $\mathrm{m} / \mathrm{z} 464.1689$ calcd. for $\mathrm{C}_{23} \mathrm{H}_{32} \mathrm{O}_{6} \mathrm{SSi}$, found 464.1672 for $[\mathrm{M}]^{-}$.

Compound 12:

Amorphous, $[\alpha]_{20}^{\mathrm{D}}=+40.4(\mathrm{c}=0.1$ in methanol);

${ }^{1} \mathrm{H}$ NMR (600 MHz, DMSO- $d_{6}$, HMBC-HSQC, COSY, NOESY) $\delta 7.67-7.62(\mathrm{~m}, 4 \mathrm{H}, \mathrm{Ph}) 7.46-7.43$ $(\mathrm{m}, 2 \mathrm{H}, \mathrm{Ph}), 7.42-7.39(\mathrm{~m}, 4 \mathrm{H}, \mathrm{Ph}), 5.30\left(\mathrm{~d}, J_{3,3-\mathrm{OH}}=\right.$ $4.0 \mathrm{~Hz}, 1 \mathrm{H}, 3-\mathrm{OH}), 4.60\left(\mathrm{dd}, J_{3,4}=2.8 \mathrm{~Hz}, J_{4,5}=9.0\right.$ $\mathrm{Hz}, 1 \mathrm{H}, \mathrm{H}-4), 4.24$ (ddd $\approx$ br. s, $1 \mathrm{H}, \mathrm{H}-3$ ), $3.82-$ 3.75 (m, 3H, H-5, H-6 $\left.{ }^{\mathrm{a}}, \mathrm{H}-6 \mathrm{~b}\right), 3.68$ (ddd $\approx \mathrm{dt}, J_{\text {lax,leq }}$ $=11.2 \mathrm{~Hz}, J_{1 \mathrm{ax}, 2 \mathrm{ax}}=11.6 \mathrm{~Hz}, J_{1 \mathrm{ax}, 2 \mathrm{eq}}=2.2 \mathrm{~Hz}, 1 \mathrm{H}$, $\mathrm{H}-1 \mathrm{ax}), 3.62\left(\mathrm{ddd}, J_{1 \mathrm{ax}, \text { leq }}=11.2 \mathrm{~Hz}, J_{\text {leq, } 2 \mathrm{ax}}=5.2 \mathrm{~Hz}\right.$, $\left.J_{\text {leq,2eq }}=2.1 \mathrm{~Hz}, 1 \mathrm{H}, \mathrm{H}-1 \mathrm{eq}\right), 3.16\left(\mathrm{~s}, 3 \mathrm{H}, \mathrm{CH}_{3}\right), 1.78$ (dddd, $J_{2 \mathrm{ax}, 3}=2.2 \mathrm{~Hz}, 1 \mathrm{H}, \mathrm{H}-2 \mathrm{ax}$ ), 1.68 (dddd $\approx$ ddt, $\left.J_{\text {2ax }, \text { eq }}=13.8 \mathrm{~Hz}, J_{2 \mathrm{eq}, 3}=4.3 \mathrm{~Hz}, 1 \mathrm{H}, \mathrm{H}-2 \mathrm{eq}\right), 0.98(\mathrm{~s}$, $9 \mathrm{H}, \mathrm{tBu})$;

${ }^{1} \mathrm{H}$ NMR (600 MHz, $\mathrm{CDCl}_{3}$, HSQC-DEPT) $\delta 7.69-$ $7.66(\mathrm{~m}, 4 \mathrm{H}, \mathrm{Ph}) 7.44-7.41(\mathrm{~m}, 2 \mathrm{H}, \mathrm{Ph}), 7.39-7.36$ $(\mathrm{m}, 4 \mathrm{H}, \mathrm{Ph}), 4.84\left(\mathrm{dd}, J_{3,4}=2.8 \mathrm{~Hz}, J_{4,5}=9.3 \mathrm{~Hz}, 1 \mathrm{H}\right.$, H-4), 4.49 (ddd $\left.\approx \mathrm{q}, J_{2 \mathrm{eq}, 3}=4.3 \mathrm{~Hz}, 1 \mathrm{H}, \mathrm{H}-3\right), 3.90$ $\left(\mathrm{m}_{\mathrm{c}}, 1 \mathrm{H}, \mathrm{H}-6 \mathrm{a}\right), 3.82\left(\mathrm{ddd} \approx \mathrm{dt}, J_{1 \mathrm{ax}, 2 \mathrm{ax}}=11.8 \mathrm{~Hz}\right.$, $\left.J_{1 \mathrm{ax}, 2 \mathrm{eq}}=3.7 \mathrm{~Hz}, 1 \mathrm{H}, \mathrm{H}-1 \mathrm{ax}\right), 3.80-3.77(\mathrm{~m}, 2 \mathrm{H}, \mathrm{H}-$ 5 , H-6b), 3.73 (ddd, $J_{1 \mathrm{ax}, 1 \mathrm{eq}}=11.4 \mathrm{~Hz}, J_{\text {leq, } 2 \mathrm{ax}}=5.0$ $\left.\mathrm{Hz}, J_{\text {leq,2eq }}=1.9 \mathrm{~Hz}, 1 \mathrm{H}, \mathrm{H}-1 \mathrm{eq}\right), 2.92\left(\mathrm{~s}, 3 \mathrm{H}, \mathrm{CH}_{3}\right)$, 2.34 (br. s, 1H, 3-OH), 1.95 (dddd, $J_{1 \mathrm{ax}, 2 \mathrm{ax}}=11.9 \mathrm{~Hz}$, $\left.J_{2 \mathrm{ax}, 3}=2.7 \mathrm{~Hz}, 1 \mathrm{H}, \mathrm{H}-2 \mathrm{ax}\right), 1.90$ (dddd $\approx \mathrm{ddt}, J_{2 \mathrm{ax}, 2 \mathrm{eq}}$ $\left.=14.3 \mathrm{~Hz}, J_{2 \mathrm{eq}, 3}=4.3 \mathrm{~Hz}, 1 \mathrm{H}, \mathrm{H}-2 \mathrm{eq}\right), 1.09$ (s, 9H, $t \mathrm{Bu}$;

${ }^{13} \mathrm{C}$ NMR (75 MHz, DMSO-d $\left.d_{6}\right) 135.3(2 \mathrm{C}, o \mathrm{Ph})$, $135.2(2 \mathrm{C}, o \mathrm{Ph}), 133.1$ (Si-CAr), 132.9 (Si-CAr), $129.9(2 \mathrm{C}, p \mathrm{Ph}), 127.9(2 \mathrm{C}, m \mathrm{Ph}), 127.8(2 \mathrm{C}, m \mathrm{Ph})$, 77.3 (C-4), 73.6 (C-5), 63.8 (C-3), 63.1 (C-6), 60.0 $(\mathrm{C}-1), 38.6\left(\mathrm{SO}_{2} \mathrm{CH}_{3}\right), 32.7(\mathrm{C}-2), 26.7\left(3 \mathrm{C}, \mathrm{CH}_{3}\right)$, 19.0 (Si-C);

${ }^{13} \mathrm{C}$ NMR $\left(151 \mathrm{MHz}, \mathrm{CDCl}_{3}\right) \delta 136.1(2 \mathrm{C}, o \mathrm{Ph})$, 135.7 (2C, oPh), 133.8 (Si-CAr), 133.1 (Si-CAr), $129.92(p \mathrm{Ph}), 129.90(p \mathrm{Ph}), 127.9(2 \mathrm{C}, m \mathrm{Ph}), 127.8$ (2C, $m \mathrm{Ph}), 77.9(\mathrm{C}-4), 73.6(\mathrm{C}-5), 66.3(\mathrm{C}-3), 63.2$ (C-6), 61.8 (C-1), $38.4\left(\mathrm{SO}_{2} \mathrm{CH}_{3}\right), 32.1$ (C-2), 27.0 (3C, $\left.\mathrm{CH}_{3}\right), 19.5(\mathrm{Si}-\mathrm{C})$;

$\left.\begin{array}{llllll}M S & (\mathrm{CI}, & \left.\mathrm{NH}_{3}\right) & m / z & 482.2 & {\left[\mathrm{M}+\mathrm{NH}_{4}\right.}\end{array}\right]^{+}$. 
Compound 13:

${ }^{1} \mathrm{H}$ NMR (300 MHz, DMSO-d $\left.\mathrm{d}_{6}, \mathrm{HSQC}, \mathrm{HMBC}\right)$ $\delta 7.66-7.63(\mathrm{~m}, 4 \mathrm{H}, o \mathrm{Ph}), 7.50-7.40(\mathrm{~m}, 6 \mathrm{H}, m \mathrm{Ph}$, $p \mathrm{Ph}$ ), 3.98 (ddd $\left.\approx \mathrm{dt}, J_{4,5}=1.7 \mathrm{~Hz}, 1 \mathrm{H}, \mathrm{H}-5\right), 3.74$ $\left(\mathrm{dd}, J_{5,6 \mathrm{a}}=6.4 \mathrm{~Hz}, J_{6 \mathrm{a}, 6 \mathrm{~b}}=10.0 \mathrm{~Hz}, 1 \mathrm{H}, \mathrm{H}-6 \mathrm{a}\right), 3.64$ $\left(\mathrm{dd}, J_{6 \mathrm{a}, 6 \mathrm{~b}}=10.0 \mathrm{~Hz}, J_{5,6 \mathrm{~b}}=6.7 \mathrm{~Hz}, 1 \mathrm{H}, \mathrm{H}-6 \mathrm{~b}\right), 3.50$ $\left(\mathrm{ddd} \approx \mathrm{dt}, J_{\text {leq, 1ax }}=11.5 \mathrm{~Hz}, J_{\text {leq, } 2 \mathrm{ax}}=5.1 \mathrm{~Hz}, 1 \mathrm{H}\right.$, $\mathrm{H}-1 \mathrm{eq}), 3.38$ (ddd $\approx \mathrm{dt}, J_{2 \mathrm{ax}, 3}=4.1 \mathrm{~Hz}, J_{2 \mathrm{eq}, 3}=1.6$ $\mathrm{Hz}, 1 \mathrm{H}, \mathrm{H}-3), 3.23$ (ddd, $J_{1 \mathrm{ax}, 1 \mathrm{eq}}=11.5 \mathrm{~Hz}, J_{1 \mathrm{ax}, 2 \mathrm{ax}}=$ $\left.8.2 \mathrm{~Hz}, J_{1 \mathrm{ax}, 2 \mathrm{eq}}=5.3 \mathrm{~Hz}, 1 \mathrm{H}, \mathrm{H}-1 \mathrm{ax}\right), 3.21\left(\mathrm{dd}, J_{3,4}=\right.$ $4.3 \mathrm{~Hz}, 1 \mathrm{H}, \mathrm{H}-4), 1.83$ (dddd $\approx \mathrm{ddt}, 2 \mathrm{H}, \mathrm{H}-2 \mathrm{ax}$ ), 1.77 $\left(\mathrm{dddd} \approx \mathrm{ddt}, J_{2 \mathrm{ax}, 2 \mathrm{eq}}=15.2 \mathrm{~Hz}, 1 \mathrm{H}, \mathrm{H}-2 \mathrm{eq}\right), 1.00$ (s, $9 \mathrm{H}, t \mathrm{Bu}) ;{ }^{1} \mathrm{H}$ NMR $(600 \mathrm{MHz}, \mathrm{CDCl} 3$, HSQCDEPT, COSY) $\delta 7.70-7.68(\mathrm{~m}, 4 \mathrm{H}, o \mathrm{Ph}), 7.44-$ $7.36(\mathrm{~m}, 6 \mathrm{H}, m \mathrm{Ph}, p \mathrm{Ph}), 3.98\left(\mathrm{ddd}, J_{4,5}=1.8 \mathrm{~Hz}, J_{5,6 \mathrm{a}}\right.$ $\left.=7.5 \mathrm{~Hz}, J_{5,6 \mathrm{~b}}=6.1 \mathrm{~Hz}, 1 \mathrm{H}, \mathrm{H}-5\right), 3.85\left(\mathrm{dd}, J_{5,6 \mathrm{a}}=\right.$ $\left.7.5 \mathrm{~Hz}, J_{6 \mathrm{a}, 6 \mathrm{~b}}=10.0 \mathrm{~Hz}, 1 \mathrm{H}, \mathrm{H}-6 \mathrm{a}\right), 3.79$ (dd, $J_{6 \mathrm{a}, 6 \mathrm{~b}}=$ $\left.10.0 \mathrm{~Hz}, J_{5,6 \mathrm{~b}}=6.7 \mathrm{~Hz}, 1 \mathrm{H}, \mathrm{H}-6 \mathrm{~b}\right), 3.65(\mathrm{ddd} \approx \mathrm{dt}$, $\left.J_{\text {leq, } 1 \mathrm{ax}}=11.6 \mathrm{~Hz}, J_{\text {leq,2eq }}=4.9 \mathrm{~Hz}, 1 \mathrm{H}, \mathrm{H}-1 \mathrm{eq}\right), 3.40$ $\left(\mathrm{ddd} \approx \mathrm{dt}, J_{2 \mathrm{ax}, 3}=0.8 \mathrm{~Hz}, J_{2 \mathrm{eq}, 3}=4.3 \mathrm{~Hz}, 1 \mathrm{H}, \mathrm{H}-3\right)$, $3.32\left(\mathrm{dd}, J_{3,4}=4.1 \mathrm{~Hz}, J_{4,5}=1.8 \mathrm{~Hz}, 1 \mathrm{H}, \mathrm{H}-4\right), 3.30$ $\left(\mathrm{ddd}, J_{1 \mathrm{ax}, 1 \mathrm{eq}}=11.6 \mathrm{~Hz}, J_{1 \mathrm{ax}, 2 \mathrm{ax}}=8.5 \mathrm{~Hz}, J_{1 \mathrm{ax}, 2 \mathrm{eq}}=4.7\right.$ Hz, 1H, H-1 ax), 1.99 (dddd $\approx$ br. ddd, $J_{\text {leq, } 2 \mathrm{ax}}=5.5$ $\mathrm{Hz}, 1 \mathrm{H}, \mathrm{H}-2 \mathrm{ax}$ ), 1.89 (dddd $\approx \mathrm{dq}, J_{2 \mathrm{ax}, 2 \mathrm{eq}}=15.2 \mathrm{~Hz}$, $1 \mathrm{H}, \mathrm{H}-2 \mathrm{eq}), 1.07$ (s, 9H, $t \mathrm{Bu}$ );

${ }^{13} \mathrm{C}$ NMR (75 MHz, DMSO-d 6 ) $\delta 135.1(2 \mathrm{C}, o \mathrm{Ph})$, $135.1 \quad(2 \mathrm{C}, \quad o \mathrm{Ph}), \quad 132.8 \quad(2 \mathrm{C}, \quad \mathrm{Si}-\mathrm{CPh}), \quad 129.9$ $(2 \mathrm{C}, p \mathrm{Ph}), 127.93(2 \mathrm{C}, m \mathrm{Ph}), 127.90(2 \mathrm{C}, m \mathrm{Ph}), 72.4$ (C-5), 63.3 (C-6), 60.1 (C-1), 49.6 (C-4), 49.2 (C-3), $38.7\left(\mathrm{SCH}_{3}\right), 26.6(3 \mathrm{C}, t \mathrm{Bu}), 23.7(\mathrm{C}-2), 18.8(\mathrm{SiC})$;

${ }^{13} \mathrm{C}$ NMR (151 MHz, $\left.\mathrm{CDCl}_{3}\right) \delta 135.7(4 \mathrm{C}, o \mathrm{Ph})$, 133.6 (Si-CPh), 133.5 (Si-CPh), 129.8 (2C, pPh), $127.9(2 \mathrm{C}, m \mathrm{Ph}), 127.8(2 \mathrm{C}, m \mathrm{Ph}), 73.3$ (C-5), 63.2 (C-6), 61.0 (C-1), 50.7 (C-4), 50.2 (C-3), 31.1 $\left(\mathrm{SCH}_{3}\right), 27.0(3 \mathrm{C}, t \mathrm{Bu}), 24.4(\mathrm{C}-2), 19.4(\mathrm{SiC})$;

MS (EI pos.) $m / z$ 368.181 calcd. for $\mathrm{C}_{22} \mathrm{H}_{28} \mathrm{O}_{3} \mathrm{Si}$, found 368.180 for $[\mathrm{M}]^{+}$.

\section{1,5-Anhydro-3-azido-6-O-tert-butyldiphenylsilyl 2,3-dideoxy-D-xylo-hexitol (14)}

To a solution of compound $11(0.77 \mathrm{~g}, 1.60 \mathrm{mmol})$ in dry DMF $(6.4 \mathrm{~mL})$ was added sodium azide $(0.52 \mathrm{~g}$, $8.04 \mathrm{mmol})$. The reaction mixture was then stirred at $90{ }^{\circ} \mathrm{C}$ for 2 days. After this time TLC (ethyl acetate/hexane 1:1) showed the complete consumption of the starting material. The reaction mixture was concentrated under reduced pressure, and the residue was taken up in ethyl acetate $(20 \mathrm{~mL})$ and washed with brine $(10 \mathrm{~mL})$ and then with water $(10 \mathrm{~mL})$, dried over magnesium sulfate, and concentrated in vacuo. The crude product was purified by column chromatography (ethyl acetate/hexane 1.8$)$ to give the azide $14(0.45 \mathrm{~g}$, $68 \%)$ as a colorless solid; $[\alpha]_{20}^{\mathrm{D}}=-2.1(\mathrm{c}=0.1$ in methanol);

${ }^{1} \mathrm{H}$ NMR (600 MHz, $\mathrm{CDCl}_{3}$, HSQC-DEPT) $\delta 7.71$ 7.67 (m, 4H, Ph), 7.46 - 7.42 (m, 2H, Ph), 7.41 - 7.38 $(\mathrm{m}, 4 \mathrm{H}, \mathrm{Ph}), 3.91(\mathrm{ddd} \approx \mathrm{q}, 1 \mathrm{H}, \mathrm{H}-3), 3.87\left(\mathrm{dd}, J_{5,6 \mathrm{a}}=\right.$ $\left.5.1 \mathrm{~Hz}, J_{6 \mathrm{a}, 6 \mathrm{~b}}=10.9 \mathrm{~Hz}, 1 \mathrm{H}, \mathrm{H}-6 \mathrm{a}\right), 3.84$ (ddd $\approx$ br. d, $1 \mathrm{H}, \mathrm{H}-1 \mathrm{eq}), 3.83$ (dd, $\left.J_{5,6 \mathrm{a}}=4.0 \mathrm{~Hz}, 1 \mathrm{H}, \mathrm{H}-6 \mathrm{~b}\right), 3.82$ $(\mathrm{m}, 1 \mathrm{H}, \mathrm{H}-5), 3.69\left(\mathrm{ddd} \approx \mathrm{dt}, J_{1 \mathrm{ax}, 1 \mathrm{eq}}=11.6 \mathrm{~Hz}\right.$, $\left.J_{\text {lax }, 2 \mathrm{eq}}=2.2 \mathrm{~Hz}, 1 \mathrm{H}, \mathrm{H}-1 \mathrm{ax}\right), 3.62(\mathrm{ddd} \approx \mathrm{dt}, 1 \mathrm{H}$,
$\mathrm{H}-4), 3.44\left(\mathrm{~d}, J_{4,4-\mathrm{OH}}=4.4 \mathrm{~Hz}, 1 \mathrm{H}, 4-\mathrm{OH}\right), 2.29$ (dddd, $J_{1 \mathrm{ax}, 2 \mathrm{ax}}=12.8 \mathrm{~Hz}, J_{1 \mathrm{eq}, 2 \mathrm{ax}}=5.2 \mathrm{~Hz}, J_{2 \mathrm{ax}, 2 \mathrm{eq}}=$ $\left.14.6 \mathrm{~Hz}, J_{2 \mathrm{ax}, 3}=3.5 \mathrm{~Hz}, 1 \mathrm{H}, \mathrm{H}-2 \mathrm{ax}\right), 1.56(\mathrm{dddd} \approx \mathrm{br}$. d, 1H, H-2eq), 1.08 (s, 9H, $t \mathrm{Bu})$;

${ }^{1} \mathrm{H}$ NMR (600 MHz, DMSO- $d_{6}$, HSQC-HMBC, COSY, NOESY) $\delta 7.65-7.62(\mathrm{~m}, 4 \mathrm{H}, o \mathrm{Ph}), 7.48-$ $7.42(\mathrm{~m}, 6 \mathrm{H}, m \mathrm{Ph}, p \mathrm{Ph}), 5.25\left(\mathrm{~d}, J_{4,4-\mathrm{OH}}=5.8 \mathrm{~Hz}\right.$, $1 \mathrm{H}, 4-\mathrm{OH}), 3.89(\mathrm{ddd} \approx \mathrm{q}, 1 \mathrm{H}, \mathrm{H}-3), 3.75\left(\mathrm{dd}, J_{5,6 \mathrm{a}}=\right.$ $\left.5.2 \mathrm{~Hz}, J_{6 \mathrm{a}, 6 \mathrm{~b}}=9.9 \mathrm{~Hz}, 1 \mathrm{H}, \mathrm{H}-6 \mathrm{a}\right), 3.67-3.61(\mathrm{~m}, 3 \mathrm{H}$, H-1eq, H-5, H-6b), 3.53 (ddd $\approx$ br. t, H-5), 3.49 (ddd $\left.\approx \mathrm{dt}, J_{1 \mathrm{ax}, 2 \mathrm{eq}}=2.6 \mathrm{~Hz}, 1 \mathrm{H}, \mathrm{H}-1 \mathrm{ax}\right), 2.06(\mathrm{dddd} \approx \mathrm{ddt}$, $\left.J_{1 \mathrm{ax}, 2 \mathrm{ax}}=11.4, J=4.6 \mathrm{~Hz}, J=3.6 \mathrm{~Hz}, 1 \mathrm{H}, \mathrm{H}-2 \mathrm{ax}\right)$, $1.43\left(\mathrm{dddd} \approx \mathrm{dq}, J_{2 \mathrm{ax}, 2 \mathrm{eq}}=14.3 \mathrm{~Hz}, 1 \mathrm{H}, \mathrm{H}-2 \mathrm{eq}\right), 0.98$ $(\mathrm{s}, 9 \mathrm{H}, t \mathrm{Bu})$;

${ }^{1} \mathrm{H}$ NMR (600 MHz, $\mathrm{C}_{6} \mathrm{D}_{6}$, HSQC, COSY, NOESY) $\delta$ 7.83-7.81 (m, 2H, Ph), $7.77-7.74(\mathrm{~m}, 2 \mathrm{H}, \mathrm{Ph})$, $7.25-7.20(\mathrm{~m}, 6 \mathrm{H}, \mathrm{Ph}), 3.87\left(\mathrm{dd}, J_{5,6 \mathrm{a}}=4.8 \mathrm{~Hz}, J_{6 \mathrm{a}, 6 \mathrm{~b}}\right.$ $=10.9 \mathrm{~Hz}, 1 \mathrm{H}, \mathrm{H}-6 \mathrm{a}), 3.71\left(\mathrm{dd}, J_{5,6 \mathrm{a}}=4.1 \mathrm{~Hz}, 1 \mathrm{H}\right.$, H-6b), 3.64 (ddd $\approx$ br. t, $1 \mathrm{H}, \mathrm{H}-4), 3.46(\mathrm{ddd} \approx \mathrm{dt}$, $\left.J_{4,5}=1.3 \mathrm{~Hz}, 1 \mathrm{H}, \mathrm{H}-5\right), 3.44$ (ddd $\approx$ br. d, $1 \mathrm{H}$, $\mathrm{H}-1$ eq),3.43 (ddd $\approx \mathrm{q}, 1 \mathrm{H}, \mathrm{H}-3), 3.41$ (ddd $\approx \mathrm{dt}$, $\left.J_{1 \mathrm{ax}, \text { leq }}=11.6 \mathrm{~Hz}, J_{1 \mathrm{ax}, 2 \mathrm{eq}}=2.2 \mathrm{~Hz}, 1 \mathrm{H}, \mathrm{H}-1 \mathrm{ax}\right), 3.18$ $\left(\mathrm{d}, J_{4,4-\mathrm{OH}}=4.8 \mathrm{~Hz}, 1 \mathrm{H}, 4-\mathrm{OH}\right), 1.90$ (dddd, $J_{1 \mathrm{ax}, 2 \mathrm{ax}}=$ $\left.12.2 \mathrm{~Hz}, J_{\text {leq,2ax }}=5.6 \mathrm{~Hz}, J_{2 \mathrm{ax}, 3}=3.4 \mathrm{~Hz}, 1 \mathrm{H}, \mathrm{H}-2 \mathrm{ax}\right)$, $0.95\left(\mathrm{dd}, J_{2 \mathrm{ax}, 2 \mathrm{eq}}=14.4 \mathrm{~Hz}, 1 \mathrm{H}, \mathrm{H}-2 \mathrm{eq}\right), 1.13$ (s, 9H, $t \mathrm{Bu})$;

${ }^{13} \mathrm{C}$ NMR (151 MHz, $\left.\mathrm{CDCl}_{3}\right) \delta 135.8(2 \mathrm{C}, o \mathrm{Ph})$, $135.7(2 \mathrm{C}, o \mathrm{Ph}), 132.9$ (Si-CAr), 132.7 (Si-CAr), $130.07(p \mathrm{Ph}), 130.04(p \mathrm{Ph}), 127.98(2 \mathrm{C}, m \mathrm{Ph})$, $127.94(2 \mathrm{C}, m \mathrm{Ph}), 73.7$ (C-4), 68.1 (C-5), 65.3 (C-6), 63.3 (C-1), 58.9 (C-3), 26.9 (3C, $t \mathrm{Bu}), 25.0$ (C-2), $19.3(\mathrm{SiC})$;

${ }^{13} \mathrm{C}$ NMR $\left(151 \mathrm{MHz}, \mathrm{DMSO}-d_{6}\right) \delta 135.14(2 \mathrm{C}, o \mathrm{Ph})$, 135.12 (2C, oPh), 133.12 (Si-CAr), 133.06 (Si-CAr), $129.93(p \mathrm{Ph}), 129.92(p \mathrm{Ph}), 128.0(4 \mathrm{C}, m \mathrm{Ph}), 75.5$ (C-5), 65.4 (C-4), 62.9 (C-6), 61.6 (C-1), 58.9 (C-3), $26.6(t \mathrm{Bu}), 25.2(\mathrm{C}-2), 18.8(\mathrm{SiC})$;

HRMS (GC TOF) $\mathrm{m} / \mathrm{z} 412.19705$ calcd. for $\mathrm{C}_{22} \mathrm{H}_{30} \mathrm{~N}_{3} \mathrm{O}_{3} \mathrm{Si}$, found 412.20509 for $\left[\mathrm{MH}^{+}\right], \mathrm{m} / \mathrm{z}$ 384.19978 calcd. for $\mathrm{C}_{22} \mathrm{H}_{30} \mathrm{NO}_{3} \mathrm{Si}$, found 384.19895 $\left[\mathrm{MH}^{+}-\mathrm{N}_{2}\right]^{+}$.

\section{1,5-Anhydro-3-azido-2,3-dideoxy-D-xylo-hexitol \\ (15)}

To a solution of compound $14(0.77 \mathrm{~g}, 1.60 \mathrm{mmol})$ in dry THF $(3 \mathrm{~mL})$ was added a $1 \mathrm{M}$ solution of tetrabutylammonium fluoride $(1.2 \mathrm{~mL}, 1.2 \mathrm{eq}$.). The reaction mixture was the stirred at $\mathrm{rt}$ for $1 \mathrm{~h}$. After this time TLC (ethyl acetate) showed the complete consumption of the starting material. The reaction mixture was concentrated under reduced pressure. The residue was taken up in ethyl acetate $(15 \mathrm{~mL})$ and washed with water $(10 \mathrm{~mL})$, dried over magnesium sulfate, and concentrated in vacuo. The crude product was purified by column chromatography (ethyl acetate/hexane 1:1) to give compound $15(0.16 \mathrm{~g}, 90 \%)$ as a colourless syrup; $[\alpha]_{20}^{\mathrm{D}}=+46.3(\mathrm{c}=0.1$ in methanol);

${ }^{1} \mathrm{H}$ NMR (600 MHz, $\mathrm{CDCl}_{3}$, HSQC-DEPT) $\delta 3.90$ $\left(\mathrm{dd}, J_{5,6 \mathrm{a}}=3.7 \mathrm{~Hz}, J_{6 \mathrm{a}, 6 \mathrm{~b}}=12.0 \mathrm{~Hz}, 1 \mathrm{H}, \mathrm{H}-6 \mathrm{a}\right), 3.89$ (m, 1H, H-3), 3.88 (ddd $\approx$ br. d, 1H, H-1eq), 3.85 $\left(\mathrm{dd}, J_{5,6 \mathrm{a}}=4.4 \mathrm{~Hz}, 1 \mathrm{H}, \mathrm{H}-6 \mathrm{~b}\right), 3.77$ (ddd $\approx \mathrm{dt}, J_{1 \mathrm{ax}, 1 \mathrm{eq}}$ 
$\left.=11.8 \mathrm{~Hz}, J_{1 \mathrm{ax}, 2 \mathrm{eq}}=2.3 \mathrm{~Hz}, 1 \mathrm{H}, \mathrm{H}-1 \mathrm{ax}\right), 3.73(\mathrm{ddd} \approx$ br. d, $\left.J_{3,4}=3.3 \mathrm{~Hz}, 1 \mathrm{H}, \mathrm{H}-4\right), 3.66\left(\mathrm{ddd} \approx \mathrm{dt}, J_{4,5}=\right.$ $1.2 \mathrm{~Hz}, 1 \mathrm{H}, \mathrm{H}-5), 3.48$ (s, 1H, 4-OH), 2.28 (dddd, $J_{1 \mathrm{ax}, 2 \mathrm{ax}}=12.7 \mathrm{~Hz}, J_{1 \mathrm{eq}, 2 \mathrm{ax}}=3.4 \mathrm{~Hz}, J_{2 \mathrm{ax}, 2 \mathrm{eq}}=14.6 \mathrm{~Hz}$, $\left.J_{2 \mathrm{ax}, 3}=5.2 \mathrm{~Hz}, 1 \mathrm{H}, \mathrm{H}-2 \mathrm{ax}\right), 1.60(\mathrm{dddd} \approx \mathrm{dq}, 1 \mathrm{H}, \mathrm{H}-$ 2eq);

${ }^{13} \mathrm{C}$ NMR (151 MHz, $\left.\mathrm{CDCl}_{3}\right) \delta 73.6$ (C-5), 69.0 (C-4), 64.6 (C-6), 63.2 (C-1), 58.9 (C-3), 24.9 (C-2); ${ }^{1} \mathrm{H}$ NMR (300 MHz, DMSO- $d_{6}, \mathrm{HMBC}$, HSQC, COSY, NOESY) $\delta 5.14\left(\mathrm{~d}, J_{4,4-\mathrm{OH}}=5.9 \mathrm{~Hz}, 1 \mathrm{H}\right.$, 4-OH), 4.58-4.55 (m, 1H, 6-OH), 3.86 (ddd $\approx$ br. q, $\Sigma \mathrm{J}=11.4 \mathrm{~Hz}, 1 \mathrm{H}, \mathrm{H}-4), 3.66\left(\mathrm{ddd}, J_{1 \mathrm{ax}, \text { leq }}=11.5 \mathrm{~Hz}\right.$, $J_{\text {leq,2ax }}=4.8 \mathrm{~Hz}, J_{1 \text { eq,2eq }}=2.9 \mathrm{~Hz}, 1 \mathrm{H}, \mathrm{H}-1$ eq $), 3.52-$ 3.38 (m, 5H, H-1ax, H-3, H-5, H-6a, H-6b), 2.06 (dddd, $J_{1 \mathrm{ax}, 2 \mathrm{ax}}=11.3 \mathrm{~Hz}, J_{1 \mathrm{eq}, 2 \mathrm{ax}}=4.8 \mathrm{~Hz}, J_{2 \mathrm{ax}, 2 \mathrm{eq}}=$ $\left.14.2 \mathrm{~Hz}, J_{2 \mathrm{ax}, 3}=3.5 \mathrm{~Hz}, 1 \mathrm{H}, \mathrm{H}-2 \mathrm{ax}\right), 1.44$ (ddddd $\approx$ br. dq, $J_{2 \mathrm{ax}, 2 \mathrm{eq}}=14.2 \mathrm{~Hz}, J_{1 \text { eq, } 2 \mathrm{eq}}=2.9 \mathrm{~Hz}, J=1.0 \mathrm{~Hz}$, $\left.\sum \mathrm{J}=24 \mathrm{~Hz}, 1 \mathrm{H}, \mathrm{H}-2 \mathrm{eq}\right)$;

${ }^{13} \mathrm{C}$ NMR (75 MHz, DMSO-d $\left.d_{6}\right) \delta 75.8(\mathrm{C}-5), 65.6$ (C-3), 61.4 (C-1), 60.2 (C-6), 59.1 (C-4), 25.3 (C-2); HRMS GC TOF) $\mathrm{m} / \mathrm{z} 173.08091$ calcd. for $\mathrm{C}_{6} \mathrm{H}_{11} \mathrm{~N}_{3} \mathrm{O}_{3}$, found 173.07949 for $\left[\mathrm{M}^{+}\right]$.

\section{1,5-Anhydro-3-azido-2,3-dideoxy-D (-xylo- hexonic Acid (16)}

A solution of compound $15(0.183 \mathrm{~g}, 1.06 \mathrm{mmol})$ in a mixture of dichloromethane $(9 \mathrm{~mL})$ and water $(3$ $\mathrm{mL}$ ) was stirred in the absence of light together with tetrabutylammonium iodide $(0.02 \mathrm{~g}, 0.04 \mathrm{mmol})$, TEMPO $(0.03 \mathrm{~g}, \quad 0.21 \mathrm{mmol})$ and (diacetoxyiodo)benzene (BAIB, $0.85 \mathrm{~g}, 2.65 \mathrm{mmol}$ ) for $2 \mathrm{~h}$ at $\mathrm{rt}$ (TLC AcOEt). A saturated aqueous solution of sodium sulfite was added until the mixture decolourized, and it was extracted with EtOAc $(5 \mathrm{~mL})$. The organic layer was dried with anhydrous $\mathrm{Na}_{2} \mathrm{SO}_{4}$, filtered and evaporated in vacuo. To a solution of the crude aldehyde product in $t$-BuOH $(5 \mathrm{~mL}), 2$-methyl-2-butene $(0.8 \mathrm{~mL}$, $7.4 \mathrm{mmol})$ and a solution of $\mathrm{NaClO}_{2}(0.16 \mathrm{~g}, 1.38$ mmol, $80 \%)$ and $\mathrm{NaH}_{2} \mathrm{PO}_{4} \cdot \mathrm{H}_{2} \mathrm{O}(0.21 \mathrm{~g}, 1.38 \mathrm{mmol})$ in $\mathrm{H}_{2} \mathrm{O}(5 \mathrm{~mL})$ were added, and the mixture was stirred at $\mathrm{rt}$ for $1 \mathrm{~h}$. The mixture was acidified with $10 \% \mathrm{HCl}$ aq. and extracted with EtOAc $(5 \mathrm{~mL})$. The organic layer was dried with anhydrous $\mathrm{Na}_{2} \mathrm{SO}_{4}$, filtered and evaporated in vacuo to give the crude acid which was purified by flash column chromatography $\left(\mathrm{CH}_{2} \mathrm{Cl}_{2} / \mathrm{MeOH}\right.$ 6:1 to $\left.\mathrm{MeOH}\right)$. Pure compound 16 (0.06 g, $0.32 \mathrm{mmol}, 30 \%)$ was obtained as a colorless solid; $[\alpha]_{20}^{\mathrm{D}}=+49.0(\mathrm{c}=1.0$ in methanol);

${ }^{1} \mathrm{H}$ NMR (600 MHz, DMSO- $d_{6}$, HSQC-HMBC, COSY, NOESY) $\delta 5.51$ (br. s, $1 \mathrm{H}, 4-\mathrm{OH}), 4.07$ $\left(\mathrm{d}, J_{4,5}=2.3 \mathrm{~Hz}, 1 \mathrm{H}, \mathrm{H}-5\right), 3.94\left(\mathrm{ddd} \approx \mathrm{q}, J_{2 \mathrm{eq}, 3}=3.5\right.$ $\mathrm{Hz}, 1 \mathrm{H}, \mathrm{H}-3$ ), 3.77 (ddd, $J_{1 \mathrm{ax}, \text { leq }}=11.5 \mathrm{~Hz}, 1 \mathrm{H}$, $\mathrm{H}-1$ eq $), 3.69\left(\mathrm{dd} \approx \mathrm{t}, J_{3,4}=3.4 \mathrm{~Hz}, 1 \mathrm{H}, \mathrm{H}-4\right), 3.53$ $\left(\mathrm{ddd} \approx \mathrm{dt}, J_{1 \mathrm{ax}, 1 \mathrm{eq}}=11.6 \mathrm{~Hz}, J_{1 \mathrm{ax}, 2 \mathrm{eq}}=2.4 \mathrm{~Hz}, 1 \mathrm{H}, \mathrm{H}-\right.$ $1 \mathrm{ax}$ ), 2.10 (dddd, $J_{1 \mathrm{ax}, 2 \mathrm{ax}}=11.4, J_{\text {leq, } 2 \mathrm{ax}}=3.8 \mathrm{~Hz}$, $\left.J_{2 \mathrm{ax}, 3}=4.6 \mathrm{~Hz}, 1 \mathrm{H}, \mathrm{H}-2 \mathrm{ax}\right), 1.46\left(\mathrm{dddd} \approx \mathrm{dq}, J_{1 \mathrm{eq}, 2 \mathrm{eq}}=\right.$ $\left.2.6 \mathrm{~Hz}, J_{2 \mathrm{ax}, 2 \mathrm{eq}}=14.3 \mathrm{~Hz}, 1 \mathrm{H}, \mathrm{H}-2 \mathrm{eq}\right)$;

${ }^{13} \mathrm{C}$ NMR (151 MHz, DMSO-d $\left.d_{6}\right) \delta 170.7(\mathrm{C}=\mathrm{O})$, 74.3 (C-5), 66.8 (C-4), 61.4 (C-1), 58. 3, H-4); 3.87 $\left(\mathrm{ddd}, J_{\text {leq,2eq }}=1.9 \mathrm{~Hz}, 1 \mathrm{H}, \mathrm{H}-1 \mathrm{eq}\right), 3.73(\mathrm{ddd} \approx \mathrm{dt}$, $\left.J_{1 \mathrm{ax}, \text { leq }}=11.5 \mathrm{~Hz}, J_{1 \mathrm{ax}, 2 \mathrm{eq}}=2.2 \mathrm{~Hz}, 1 \mathrm{H}, \mathrm{H}-1 \mathrm{ax}\right), 2.26$ (dddd, $J_{1 \mathrm{ax}, 2 \mathrm{ax}}=12.2, J_{1 \mathrm{eq}, 2 \mathrm{ax}}=3.3 \mathrm{~Hz}, J_{2 \mathrm{ax}, 3}=5.0 \mathrm{~Hz}$, $1 \mathrm{H}, \mathrm{H}-2 \mathrm{ax}$ ), 1.59 (dddd $\approx$ br. d, $J_{2 \mathrm{ax}, 2 \mathrm{eq}}=14.5 \mathrm{~Hz}, 1 \mathrm{H}$, H-2eq);

${ }^{13} \mathrm{C}$ NMR (151 MHz, CD $\left.{ }_{3} \mathrm{OD}\right) \delta 173.2(\mathrm{C}=\mathrm{O}), 75.7$ (C-5), 68.3 (C-48 (C-3), 24.5 (C-2);

${ }^{1} \mathrm{H}$ NMR $\left(600 \mathrm{MHz}, \mathrm{CD}_{3} \mathrm{OD}\right) \delta 4.24\left(\mathrm{~d}, J_{4,5}=1.8\right.$ $\mathrm{Hz}, 1 \mathrm{H}, \mathrm{H}-5), 3.92-3.88$ (m, 2H, H-), 63.4 (C-1), 60.6 (C-3), 25.6 (C-2).

\section{References}

1- (a) M. A. Clark, Privileged scaffolds for library design and drug discovery, Curr. Opin. Chem. Biol. 2010, 14, 396-403; (b) R. E. Kleiner, C. E. Dumelin, D. R. Liu, Small-molecule discovery from DNA-encoded chemical libraries, Chem. Soc. Rev. 2011, 40, 5707-5717; (c) R. A. Goodnow Jr., In Handbook for DNA-Encoded Chemistry: A Brief History of the Development of Combinatorial Chemistry and the Emerging Need for DNA-Encoded Chemistry; ed. by R. A.Goodnow Jr.; Wiley \& Sons: Hoboken, 2014, pp. 19-44; (d) R. M. Franzini, D. Neri, J. Scheuermann, DNA-Encoded Chemical Libraries: Advancing beyond Conventional Small-Molecule Libraries, Acc. Chem. Res. 2014, 47, 1247-1255; (e) C. Zambaldo, S. Barluenga, N. Winssinger, PNA-encoded chemical libraries, Curr. Opin. Chem. Biol. 2015, 26, 8-15; (f) G. Li, W. Zheng, Y. Liu, X. Li, Curr. Opin. Chem. Biol. 2015, 26, 25-33; (g) A. I. Chan, L. M. McGregor, D. R. Liu, Novel selection methods for DNA-encoded chemical libraries, Curr. Opin. Chem. Biol. 2015, 26, 55-61; (h) Y. Li, E. Gabriele, F. Samain, N. Favalli, F. Sladojevich, J. Scheuermann, D. Neri, Optimized Reaction Conditions for Amide Bond Formation in DNA-Encoded Combinatorial Libraries, ACS Comb. Sci. 2016, 18, 438-443; (i) H. Salamon, M. Klika Škopić, K. Jung, O. Bugain, A. Brunschweiger, Chemical Biology Probes from Advanced DNA-encoded Libraries, ACS Chem. Biol. 2016, 11, 296-307; (j) R. A. Goodnow, C. E. Dumelin, A. D. Keefe, DNA-encoded chemistry: enabling the deeper sampling of chemical space, Nat. Rev. Drug Discov. 2017, 16, 131-147; (k) A. L. Satz, What Do You Get from DNA-Encoded Libraries? , ACS Med. Chem. Lett. 2018, 9, 408-410.

2- H. Deng, H. O'Keefe, C. P. Davie, K. E. Lind, R. A. Acharya, G. J. Franklin, J. Larkin, R. Matico, M. Neeb, M. M. Thompson, T. Lohr, J. W. Gross, P. A. Centrella, G. K. O’Donovan, K. L. Bedard, K. van Vloten, S. Mataruse, S. R. Skinner, S. L. Belyanskaya, T. Y. Carpenter, T. W. Shearer, M. A. Clark, J. W. Cuozzo, C. C. Arico-Muendel, B. A. Morgan, Discovery of Highly Potent and Selective Small Molecule ADAMTS-5 Inhibitors That Inhibit Human Cartilage Degradation via Encoded Library 
Technology (ELT), J. Med. Chem. 2012, 55, 7061-7079.

3- R. M. Franzini, C. Randolph, Chemical Space of DNA-Encoded Libraries, J. Med. Chem. 2016, 59, 6629-6644.

4- (a) M. A. Clark, R. A. Acharya, C. C. AricoMuendel, S. L. Belyanskaya, D. R. Benjamin, N. R. Carlson, P. A. Centrella, C. H. Chiu, S. P. Creaser, J. W. Cuozzo, C. P. Davie, Y. Ding, G. J. Franklin, K. D. Franzen, M. L. Gefter, S. P. Hale, N. J. V. Hansen, D. I. Israel, J. Jiang, M. J. Kavarana, M. S. Kelley, C. S. Kollmann, F. Li, K. Lind, S. Mataruse, P. F. Medeiros, J. A. Messer, P. Myers, H. O'Keefe, M. C. Oliff, C. E. Rise, A. L. Satz, S. R. Skinner, J. L. Svendsen, L. Tang, K. van Vloten, R. W. Wagner, G. Yao, B. Zhao, B. A. Morgan, Design, synthesis and selection of DNA-encoded small-molecule libraries, Nat. Chem. Biol. 2009, 5, 647; (b) C. S. Kollmann, X. Bai, C.-H. Tsai, H. Yang, K. E. Lind, S. R. Skinner, Z. Zhu, D. I. Israel, J. W. Cuozzo, B. A. Morgan, K. Yuki, C. Xie, T. A. Springer, M. Shimaoka, G. Evindar, Application of encoded library technology (ELT) to a proteinprotein interaction target: discovery of a potent class of integrin lymphocyte function-associated antigen 1 (LFA-1) antagonists, Bioorg. Med. Chem. 2014, 22, 2353-2365.

5- F. Buller, M. Steiner, K. Frey, D. Mircsof, J. Scheuermann, M. Kalisch, P. Bühlmann, C. T. Supuran, D. Neri, Selection of Carbonic Anhydrase IX Inhibitors from One Million DNAEncoded Compounds, ACS Chem. Biol. 2011, 6, 336-344.

6- L. Encinas, H. O’Keefe, M. Neu, M. J. Remuiñán, A. M. Patel, A. Guardia, C. P. Davie, N. Pérez-Macías, H. Yang, M. A. Convery, J. A. Messer, E. Pérez-Herrán, P. A. Centrella, D. Álvarez-Gómez, M. A. Clark, S. Huss, G. K. O’Donovan, F. Ortega-Muro, W. McDowell, P. Castañeda, C. C. Arico-Muendel, S. Pajk, J. Rullás, I. Angulo-Barturen, E. Álvarez-Ruíz, A. Mendoza-Losana, L. Ballell Pages, J. CastroPichel, G. Evindar, Encoded Library Technology as a Source of Hits for the Discovery and Lead Optimization of a Potent and Selective Class of Bactericidal Direct Inhibitors of Mycobacterium tuberculosis InhA, J. Med. Chem. 2014, 57, 1276-1288.

7- A. M. Estévez, F. Gruber, A. L. Satz, R. E. Martin, H. P. Wessel, A carbohydrate-derived trifunctional scaffold for DNA-encoded libraries, Tetrahedron Asymm. 2017, 28, 837-842.

8- V. P. Pathak, A Convenient Method for ODeacetylation Using IRA-400(OH) Resin. Synth. Commun. 1993, 23, 83-85.

9- N. Hayashi, K. Yamada, O. Arikita, Practical and Catalytic Synthesis of 1,5-Anhydrohex-1-en-3uloses, Tetrahedron 1999, 8331-8340.

10-G. Descotes, J. C. Martin, D. Sinou, T.-C. Dung, Transfert catalytique d'hydrogène. III. Emploi de sucres insaturés, Bull. Soc. Chim. France 1979, 61-64.

11-T. Fujiwara, M. Hayashi, Efficient Synthesis of Rare Sugar d-Allal via Reversal of Diastereoselection in the Reduction of Protected 1,5-Anhydrohex-1-en-3-uloses: Protecting Group Dependence of the Stereoselection, J. Org. Chem. 2008, 73, 9161-9163.

12-T. E. Goodwin, C. M. Crowder, R. B. White, J. S. Swanson, F. E. Evans, W. L. Meyer, Stereoselective addition of organocopper reagents to a novel carbohydrate-derived 2,3dihydro-4H-pyran-4-one, J. Org. Chem. 1983, 48, 376-380.

13-T. Nguyen Dinh, D. Do Khac, I. Gandolfi, Y. Memoria, M. Fétizon, T. Prange, Réarrangements acido-catalysés des oxabicylco[4.2.0]oct-7-én-5-ones, Bull. Soc. Chim. Fr. 1993, 130, 287-298.

14-N. Hayashi, K. Fujiwara, A. Murai, Equilibrium in Ring Expansion Reactions of the Bromo Epoxides, Synlett 1997, 1997, 793-794.

15-K. Michael, H. Kessler, Michael-type additions in the synthesis of $\alpha-\mathrm{O}$ - and -S-2deoxyglycosides, Tetrahedron Lett. 1996, 37 , 3453-3456.

16-I. Paterson, O. Delgado, G. J. Florence, I. Lyothier, M. O'Brien, J. P. Scott, N. Sereinig, A Second-Generation Total Synthesis of (+)Discodermolide: The Development of a Practical Route Using Solely Substrate-Based Stereocontrol, J. Org. Chem. 2005, 70, 150-160.

17-Crystallographic data for the structure in this paper have been deposited with the Cambridge Crystallographic Data Centre as supplementary publication numbers CCDC 1836178. This data can be obtained, free of charge, on application to CCDC, 12 Union Road, Cambridge CB2 1EZ, UK [fax: $+44(0)-1223-336033$ or e-mail: deposit@ ccdc.cam.ac.uk]. 